\title{
Spin and Pseudospin Symmetry in Generalized Manning-Rosen Potential
}

\author{
Hilmi Yanar and Ali Havare \\ Department of Physics, Mersin University, 33143 Mersin, Turkey \\ Correspondence should be addressed to Hilmi Yanar; hlmyanar@gmail.com
}

Received 8 May 2015; Accepted 18 June 2015

Academic Editor: Shi-Hai Dong

Copyright ( $) 2015$ H. Yanar and A. Havare. This is an open access article distributed under the Creative Commons Attribution License, which permits unrestricted use, distribution, and reproduction in any medium, provided the original work is properly cited. The publication of this article was funded by $\mathrm{SCOAP}^{3}$.

\begin{abstract}
Spin and pseudospin symmetric Dirac spinors and energy relations are obtained by solving the Dirac equation with centrifugal term for a new suggested generalized Manning-Rosen potential which includes the potentials describing the nuclear and molecular structures. To solve the Dirac equation the Nikiforov-Uvarov method is used and also applied the Pekeris approximation to the centrifugal term. Energy eigenvalues for bound states are found numerically in the case of spin and pseudospin symmetry. Besides, the data attained in the present study are compared with the results obtained in the previous studies and it is seen that our data are consistent with the earlier ones.
\end{abstract}

\section{Introduction}

Spin and pseudospin symmetries are symmetries of the Dirac Hamiltonian. Spin symmetry leads to degeneracy between two states with quantum numbers $(n, l, j=l-s)$ and $(n, l, j=l+s)$. These two states are considered as a spin doublet with $(n, l, j=l \mp s) . n, l, j$, and $s$ are radial, orbital angular momentum, total angular momentum, and spin quantum numbers, respectively. The spin doublet or spin symmetry is used to explain the spectrum of antinucleon in a nucleus [1-4] and small spin-orbit splitting in hadrons [5]. Pseudospin symmetry causes degeneracy between two states with quantum numbers $(n, l, j=l+1 / 2)$ and $(n-$ $1, l+2, j=l+3 / 2)$. These two states are regarded as a pseudospin doublet with quantum numbers $(\widetilde{n}=n-1, \widetilde{l}=$ $l+1, \tilde{j}=\widetilde{l} \mp \widetilde{s})$, where $\widetilde{l}$ and $\widetilde{s}=1 / 2$ are pseudoorbital angular momentum and pseudospin quantum numbers, respectively $[6,7]$. The pseudospin doublet or pseudospin symmetry is used to explain deformation [8], identical bands [9-11], magnetic moment [12-14], and effective shell-model [15] in the nuclear structures.

Pseudospin and pseudoorbital angular momentum were introduced for the first time to explain experimental observation of quasi-degeneracy between $(n, l, j=l+1 / 2)$ and $(n-1, l+2, j=l+3 / 2)$ states of single-nucleon in nuclei $[6,7]$. Pseudospin symmetry was discussed firstly in nonrelativistic framework [6]. However, in the 1990s, Blokhin et al. found a connection between pseudospin symmetry and relativistic mean field theory [16-18]. Then Ginocchio recognized that the pseudoorbital angular momentum $\tilde{l}$ is the orbital angular momentum of lower component of the Dirac spinor [19]. He showed that the pseudospin symmetry occurs in nuclei when the sum of the scalar potential $S(r)$ and vector potential $V(r)$ is approximately equal to zero $(V(r)+$ $S(r) \sim 0)[1,2,19-22]$. Then, Meng et al. proved that the exact pseudospin symmetry exists when $d[V(r)+S(r)] / d r=0$ or $V(r)+S(r)=$ constant in the Dirac equation [23, 24]. Also they introduced that the exact spin symmetry occurs when $d[V(r)-S(r)] / d r=0$ or $V(r)-S(r)=$ constant in the Dirac equation [3]. For two symmetries, the Dirac Hamiltonian is invariant under the SU(2) algebra [25]. After these studies, in recent years, spin and pseudospin symmetric solutions of the Dirac equation have been obtained and investigated by using different methods for various external potentials [26-66]. In [26], Wei and Dong have studied the ManningRosen potential under the spin symmetry limit. Also they have examined pseudospin symmetric solutions and energy eigenvalues for the Manning-Rosen potential [27]. Moreover 
the relativistic symmetries of the Manning-Rosen potential have been investigated by considering different approximation scheme for the centrifugal term [28, 29, 32, 33].

In this paper we have considered the new suggested generalized Manning-Rosen potential [67]. The new suggested generalized Manning-Rosen potential is defined in the following form:

$$
V(r)=\left[\frac{A e^{-2 b(r-L)}}{\left(q+p e^{-b(r-L)}\right)^{2}}+\frac{B e^{-b(r-L)}}{q+p e^{-b(r-L)}}\right]
$$

Generalized Manning-Rosen potential includes some molecular potentials which are Manning-Rosen [68-73], Morse [74-77], hyperbolic Pöschl-Teller [78], q-parameter hyperbolic Pöschl-Teller [79], and Kratzer-Fues [80-82] potentials. Also it can be reduced to Woods-Saxon [35-39, 83] and Hulthen $[40-45,84]$ potentials which are used in the explanation of the nuclear structures and to the Yukawa potential that represents the interactions between nucleons in nuclei $[55-59,85]$.

Therefore, the generalized Manning-Rosen potential is important for being able to explain both nuclear and molecular structures. If the generalized Manning-Rosen potential is considered as a nuclear potential, the spin and pseudospin symmetric solutions should be examined. Also spin and pseudospin symmetry can be investigated for diatomic molecular potentials by defining the reduced mass $\mu=$ $m_{1} m_{2} /\left(m_{1}+m_{2}\right)$, where $m_{1}$ and $m_{2}$ are two nuclei masses of a diatomic molecule. In this context, it is aimed at investigating spin and pseudospin symmetric solutions for the generalized Manning-Rosen potential and at finding the bound state energy eigenvalues of the considered potential under the spin and pseudospin symmetry limits. Another aim is to examine the special cases of the generalized Manning-Rosen potential and to compare the obtained results for special cases with the results that are obtained in the previous studies and also to check up whether these results are compatible.

The scheme of paper is as follows. In Section 2, the Nikiforov-Uvarov method that we use to solve the Dirac equation is introduced briefly. In Section 3, the second-order differential equations are obtained from Dirac equation for the spin and pseudospin symmetry. In Sections 4 and 5, the spin and pseudospin symmetric solutions and bound state energy eigenvalues are found for the generalized ManningRosen potential, respectively. In Section 6, the effects of potential parameters on the energy eigenvalues are investigated. In Section 7, special cases of the generalized ManningRosen potential are introduced and energy eigenvalues are calculated for each special case in the spin and pseudospin symmetric limits. Also in the same section our energy eigenvalues are compared with the previous ones. Finally, an appraisal of the obtained results is made in Section 8 .

\section{Nikiforov-Uvarov Method}

This section has been prepared by using [86]. The NikiforovUvarov method is used to solve the second-order differential equations which must be in the following form:

$$
\frac{d^{2} U(y)}{d y^{2}}+\frac{\widetilde{\varepsilon}(y)}{\xi(y)} \frac{d U(y)}{d y}+\frac{\widetilde{\xi}(y)}{\xi^{2}(y)} U(y)=0,
$$

where $\widetilde{\mathcal{E}}(y)$ can be first-degree polynomial maximally. $\xi(y)$ and $\tilde{\xi}(y)$ can be polynomials no more than second degree. The function $U(y)$ is defined as

$$
U(y)=H(y) W(y)
$$

and the form of (2) is required to be covariant. In this case the following equation is obtained:

$$
\frac{d^{2} W(y)}{d y^{2}}+\frac{\varepsilon(y)}{\xi(y)} \frac{d W(y)}{d y}+\frac{\bar{\xi}(y)}{\xi^{2}(y)} W(y)=0,
$$

where $\varepsilon(y)$ which can be first-degree polynomials maximally and $\bar{\xi}(y)$ that can be second-degree polynomials maximally are found as follows, respectively:

$$
\begin{aligned}
\varepsilon(y)= & {\left[2 \frac{d H(y) / d y}{H(y)}+\frac{\widetilde{\varepsilon}(y)}{\xi(y)}\right] \xi(y) } \\
\Longrightarrow & \frac{d H(y) / d y}{H(y)}=\frac{\pi(y)}{\xi(y)}, \\
\bar{\xi}(y)= & \pi^{2}(y)+\widetilde{\xi}(y)+\pi(y)\left[\widetilde{\varepsilon}(y)-\frac{d \xi(y)}{d y}\right] \\
& +\xi(y) \frac{d \pi(y)}{d y}
\end{aligned}
$$

by defining the polynomial $\pi(y)$ as

$$
\pi(y)=\frac{1}{2}[\varepsilon(y)-\widetilde{\varepsilon}(y)] .
$$

The convenient $\pi(y)$ must be found to obtain solutions of (4) or (2) and to study properties of solutions. Therefore the following relation is established between $\bar{\xi}(y)$ and $\xi(y)$ :

$$
\bar{\xi}(y)=\lambda \xi(y)
$$

where $\lambda$ is a constant. In this case (4) reduces to following equation:

$$
\xi(y) \frac{d^{2} W(y)}{d y^{2}}+\varepsilon(y) \frac{d W(y)}{d y}+\lambda W(y)=0 .
$$

This is a hypergeometric type equation and solution of this equation is functions of hypergeometric type. By using (8), the polynomial $\pi(y)$ is found:

$$
\begin{aligned}
\pi(y)= & \frac{d \xi(y) / d y-\widetilde{\varepsilon}(y)}{2} \\
& \pm \sqrt{\left(\frac{d \xi(y) / d y-\widetilde{\varepsilon}(y)}{2}\right)^{2}-\tilde{\xi}(y)+k \xi(y)}
\end{aligned}
$$


where

$$
k=\lambda-\frac{d \pi(y)}{d y} .
$$

$\pi(y)$ is a first-degree polynomial. Therefore the expression in the square root in (10) must be written a square of a polynomial. Considering this property, the values are found for $k$. Derivatives of the hypergeometric functions are also the hypergeometric functions. Accordingly, $n$th derivative of $W(y)$ satisfies the hypergeometric type equation. By using this property, a relation for $\lambda$ is obtained:

$$
\lambda=-n \frac{d \varepsilon(y)}{d y}-\frac{n(n-1)}{2} \frac{d^{2} \xi(y)}{d y^{2}} .
$$

When the quantum mechanical problems are discussed, an energy relation is obtained by equating (11) to (12).

The hypergeometric type function $W(y)$ is found by using the following Rodrigues relation for hypergeometric type equations:

$$
W_{n}(y)=\frac{C_{n}}{\rho(y)} \frac{d^{n}}{d y^{n}}\left[\xi^{n}(y) \rho(y)\right],
$$

where $\rho(y)$ is a weight function and satisfies the following equation:

$$
\frac{d}{d y}[\xi(y) \rho(y)]=\varepsilon(y) \rho(y) .
$$

\section{Dirac Equation}

In the presence of an attractive scalar $S(r)$ and a repulsive vector $V(r)$ potentials, the Dirac equation for a nucleon with mass $m$ is written as follows ( $\hbar=c=1)$ [27, 46, 87]:

$$
\begin{gathered}
{[\boldsymbol{\alpha} \cdot \mathbf{p}+\beta(m+S(r))] \Psi(r, \theta, \phi)} \\
=[E-V(r)] \Psi(r, \theta, \phi),
\end{gathered}
$$

where $\boldsymbol{\alpha}$ and $\beta$ are the $4 \times 4$ Dirac matrices, while $\boldsymbol{\alpha}$ is defined in terms of $2 \times 2$ Pauli spin matrices, and $\beta$ is described in terms of $2 \times 2$ unit matrices [87]:

$$
\begin{aligned}
& \boldsymbol{\alpha}=\left(\begin{array}{cc}
0 & \boldsymbol{\sigma} \\
\boldsymbol{\sigma} & 0
\end{array}\right), \\
& \beta=\left(\begin{array}{cc}
I & 0 \\
0 & -I
\end{array}\right) .
\end{aligned}
$$

The spherically symmetric Dirac spinor wave function [1] is written as

$$
\Psi(\mathbf{r})=\left(\begin{array}{c}
\frac{F_{n \kappa}(r)}{r} Y_{j m}^{\ell}(\theta, \phi) \\
\frac{i G_{n \kappa}(r)}{r} Y_{j m}^{\tilde{\ell}}(\theta, \phi)
\end{array}\right),
$$

where $F_{n \kappa}(r)$ and $G_{n \kappa}(r)$ are the upper and lower radial wave functions, respectively. $Y_{j m}^{\ell}(\theta, \phi)$ and $Y_{j m}^{\tilde{\ell}}(\theta, \phi)$ are the spin and pseudospin spherical harmonics, respectively. $\kappa$ is the eigenvalues of spin-orbit coupling operator $K=-\beta(\boldsymbol{\sigma} \cdot \mathbf{L}+1)$. The values of $\kappa$ are $\ell$ for unaligned $\operatorname{spin}(j=\ell-1 / 2)$ and $-(\ell+1)$ for aligned spin $(j=\ell+1 / 2)$. Substituting (9) and (10) into (8) and by using the following relations [88]:

$$
\begin{aligned}
(\boldsymbol{\sigma} \cdot \mathbf{A})(\boldsymbol{\sigma} \cdot \mathbf{B}) & =\mathbf{A} \cdot \mathbf{B}+i \boldsymbol{\sigma} \cdot(\mathbf{A} \times \mathbf{B}), \\
\boldsymbol{\sigma} \cdot \mathbf{p} & =\boldsymbol{\sigma} \cdot \widehat{\mathbf{r}}\left(\widehat{\mathbf{r}} \cdot \mathbf{p}+i \frac{\boldsymbol{\sigma} \cdot \mathbf{L}}{r}\right)
\end{aligned}
$$

together with the following properties [88]:

$$
\begin{aligned}
& (\boldsymbol{\sigma} \cdot \mathbf{L}) Y_{j m}^{\tilde{\ell}}(\theta, \phi)=(\kappa-1) Y_{j m}^{\tilde{\ell}}(\theta, \phi), \\
& (\boldsymbol{\sigma} \cdot \mathbf{L}) Y_{j m}^{\ell}(\theta, \phi)=-(\kappa-1) Y_{j m}^{\ell}(\theta, \phi), \\
& (\boldsymbol{\sigma} \cdot \widehat{\mathbf{r}}) Y_{j m}^{\ell}(\theta, \phi)=-Y_{j m}^{\widetilde{\ell}}(\theta, \phi), \\
& (\boldsymbol{\sigma} \cdot \widehat{\mathbf{r}}) Y_{j m}^{\tilde{\ell}}(\theta, \phi)=-Y_{j m}^{\ell}(\theta, \phi),
\end{aligned}
$$

the Dirac equation reduces to two coupled differential equations as follows:

$$
\begin{aligned}
& \left(\frac{d}{d r}+\frac{\kappa}{r}\right) F_{n \kappa}(r)=[m+E-\Delta(r)] G_{n \kappa}(r), \\
& \left(\frac{d}{d r}-\frac{\kappa}{r}\right) G_{n \kappa}(r)=\left[m-E+\sum(r)\right] F_{n \kappa}(r),
\end{aligned}
$$

where

$$
\begin{aligned}
& \Delta(r)=V(r)-S(r), \\
& \Sigma(r)=V(r)+S(r) .
\end{aligned}
$$

From these two coupled differential equations, two secondorder differential equations have been obtained for the upper and lower radial wave functions, respectively:

$$
\begin{aligned}
& {\left[\frac{d^{2}}{d r^{2}}-\frac{\kappa(\kappa+1)}{r^{2}}+\frac{(d \Delta(r) / d r)(d / d r+\kappa / r)}{m+E-\Delta(r)}\right]} \\
& \cdot F_{n \kappa}(r)+(E+m-\Delta(r))(E-m-\Sigma(r)) F_{n \kappa}(r) \\
& \quad=0
\end{aligned}
$$




$$
\begin{aligned}
& {\left[\frac{d^{2}}{d r^{2}}-\frac{\kappa(\kappa-1)}{r^{2}}-\frac{(d \Sigma(r) / d r)(d / d r-\kappa / r)}{m-E+\Sigma(r)}\right]} \\
& \cdot G_{n \kappa}(r)+(E+m-\Delta(r))(E-m-\Sigma(r)) G_{n \kappa}(r) \\
& \quad=0
\end{aligned}
$$

where $\kappa(\kappa+1)=l(l+1)$ and $\kappa(\kappa-1)=\tilde{l}(\widetilde{l}+1)$.

\section{Spin Symmetric Solution}

In the spin symmetric case the sum of scalar potential with vector potential $\Sigma(r)$ and the difference between these potentials $\Delta(r)$ are written as follows:

$$
\begin{aligned}
& \Delta(r)=S_{s}, \\
& \Sigma(r)=\left[\frac{A e^{-2 b(r-L)}}{\left(q+p e^{-b(r-L)}\right)^{2}}+\frac{B e^{-b(r-L)}}{q+p e^{-b(r-L)}}\right],
\end{aligned}
$$

where $S_{s}$ is a constant, $L$ is the nuclear radius, $1 / b$ is the thickness of surface layer, and $A$ and $B$ are related with the potential dept for nuclei. Also $q$ and $p$ are dimensionless parameters and they are used to determine shape of the potential. Substituting (25) into (23), (23) reduces to

$$
\begin{aligned}
& \left\{\frac{d^{2}}{d r^{2}}-\frac{\kappa(\kappa+1)}{r^{2}}\right. \\
& \left.-\left(m+E-S_{s}\right)\left[\frac{A}{\left(p+q e^{b(r-L)}\right)^{2}}+\frac{B}{p+q e^{b(r-L)}}\right]\right\} \\
& \quad \cdot F_{n \kappa}(r)-(m-E)\left(m+E-S_{s}\right) F_{n \kappa}(r)=0 .
\end{aligned}
$$

To solve this equation analytically, the following approximation which is called the Pekeris approximation [89] is applied to the centrifugal term:

$$
\frac{1}{r^{2}} \approx C_{0}+\frac{C_{1}}{p+q e^{b(r-L)}}+\frac{C_{2}}{\left(p+q e^{b(r-L)}\right)^{2}},
$$

where $C_{0}, C_{1}$, and $C_{2}$ are constants and their values are found as follows by expanding the terms in (27):

$$
\begin{aligned}
& C_{0} \\
& =\frac{(3+b L) p^{2}-2(-3+b L) p q+\left(3-3 b L+b^{2} L^{2}\right) q^{2}}{b^{2} q^{2} L^{4}}, \\
& C_{1}=-\frac{2(p+q)^{2}[(3+b L) p+(3-2 b L) q]}{b^{2} q^{2} L^{4}}, \\
& C_{2}=\frac{(p+q)^{3}[(3+b L) p+(3-b L) q]}{b^{2} q^{2} L^{4}} .
\end{aligned}
$$

The terms in (27) are expanded in a series about $r \approx L$ because the nuclear distance $r$ does not fluctuate very far from the equilibrium position at $L$ [35]. Therefore the approximation in (27) gives good result only for $r \approx L$. The equivalence

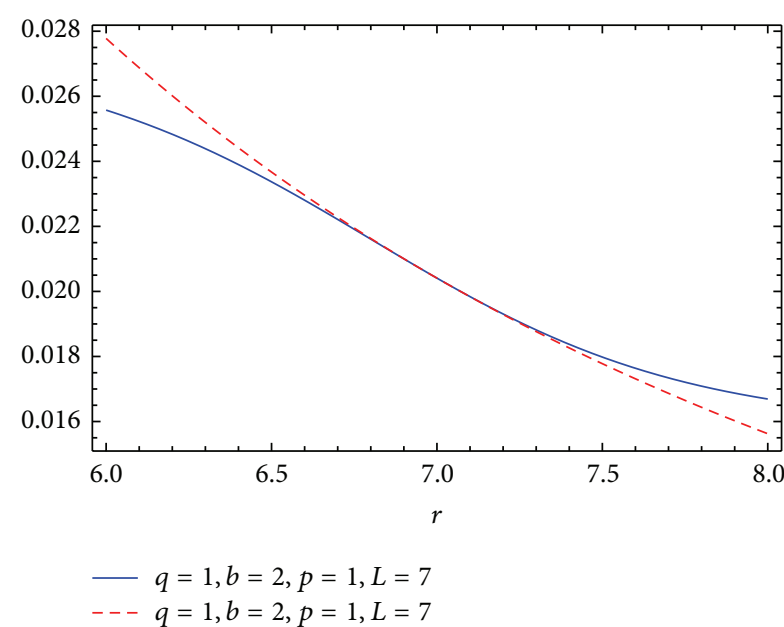

FIgURE 1: The equivalence of the Pekeris approximation to the centrifugal term.

of the Pekeris approximation to centrifugal term is shown in Figure 1. In this figure the blue curve shows the Pekeris approximation and the dotted curve indicates the centrifugal term.

Using (27) in (26) and defining variable like $z=p /(p+$ $\left.q e^{b(r-L)}\right)$, the following equation is obtained:

$$
\begin{aligned}
& \frac{d^{2} F_{n \kappa}(z)}{d z^{2}}+\frac{(1-2 z)}{z(1-z)} \frac{d F_{n \kappa}(z)}{d z} \\
& +\frac{\left(-\mu^{2}-\nu z-\eta z^{2}\right)}{z^{2}(1-z)^{2}} F_{n \kappa}(z)=0,
\end{aligned}
$$

where

$$
\begin{aligned}
\mu^{2} & =\frac{\kappa(\kappa+1) C_{0}+(m-E)\left(m+E-S_{s}\right)}{b^{2}}, \\
\nu & =\frac{\kappa(\kappa+1) C_{1}+\left(m+E-S_{s}\right) B}{p b^{2}}, \\
\eta & =\frac{\kappa(\kappa+1) C_{2}+\left(m+E-S_{s}\right) A}{p^{2} b^{2}} .
\end{aligned}
$$

Equation (29) has the same form and properties with (2). Therefore the Nikiforov-Uvarov method can be used to solve (29). By comparing this equation with (2), it is seen that

$$
\begin{aligned}
& \widetilde{\varepsilon}(z)=1-2 z, \\
& \widetilde{\xi}(z)=-\mu^{2}-\nu z-\eta z^{2}, \\
& \xi(z)=z(1-z) .
\end{aligned}
$$

Using (10) and considering the condition of polynomial $\pi(z)$ to be first-degree polynomial, the following equation is found: 


$$
\pi(z)= \begin{cases} \pm\left[\mu-\left(\mu+\sqrt{\mu^{2}+v+\eta}\right) z\right] ; & k=-\left(\nu+2 \mu^{2}\right)-2 \mu \sqrt{\mu^{2}+v+\eta} \\ \pm\left[\mu-\left(\mu-\sqrt{\mu^{2}+v+\eta}\right) z\right] ; & k=-\left(\nu+2 \mu^{2}\right)+2 \mu \sqrt{\mu^{2}+v+\eta}\end{cases}
$$

The polynomial $\pi(z)$ has four values but only one of them satisfies the physical solution. By using (5) and considering the boundary conditions $\left(r \rightarrow \infty, F_{n \kappa}(r)=0\right.$ and $r \rightarrow 0$, $\left.F_{n \kappa}(r)=0\right)$, the polynomial $\pi(z)$ which satisfies physical solution is found as follows:

$$
\begin{aligned}
& \pi(z)=\mu-\left(\mu+\sqrt{\mu^{2}+\nu+\eta}\right) z \\
& \quad \text { for } k=-\left(\nu+2 \mu^{2}\right)-2 \mu \sqrt{\mu^{2}+\nu+\eta} .
\end{aligned}
$$

In this case from (5), we obtain $H(z)$ which is a part of the upper radial wave function $F_{n \kappa}(z)$ :

$$
H(z)=z^{\mu}(1-z)^{\sqrt{\mu^{2}+\nu+\eta}} .
$$

By using (11) and (33), the following equation is found:

$$
\lambda=-\nu-2 \mu^{2}-\mu-(1+2 \mu) \sqrt{\mu^{2}+\nu+\eta}
$$

Also using (7) and (12), the following equation is attained for $\lambda$ :

$$
\lambda=2 n\left[1+\left(\mu+\sqrt{\mu^{2}+\nu+\eta}\right)\right]+n(n-1) .
$$

Equating (35) with (36), we obtain an equation that gives the bound energy values:

$$
\begin{aligned}
& E^{2}-m^{2}-S_{s}(E-m)-\kappa(\kappa+1) C_{0}-\frac{\kappa(\kappa+1) C_{1}+\left(E+m-S_{s}\right) B}{2 p}-\frac{n(n+1) b^{2}}{2}-\frac{(2 n+1) b^{2}}{2} \\
& \cdot \sqrt{\frac{\kappa(\kappa+1) C_{0}+(m-E)\left(m+E-S_{s}\right)}{b^{2}}-\frac{b^{2}}{2}\left(1+2 n+2 \sqrt{\left.\frac{\kappa(\kappa+1) C_{0}+(m-E)\left(m+E-S_{s}\right)}{b^{2}}\right)}\right.} \\
& \cdot \sqrt{\frac{\kappa(\kappa+1)\left(C_{0} p^{2}+C_{1} p+C_{2}\right)+\left(m+E-S_{s}\right)\left[A+B p+(m-E) p^{2}\right]}{b^{2} p^{2}}}=0 .
\end{aligned}
$$

It is not possible to solve this equation analytically so we use the software program to obtain energy values for bound states depending on $n, \kappa$ and the parameters of potential. Some energy eigenvalues are calculated for $q=0.8, p=0.65, b=$ $2 \mathrm{fm}^{-1}, L=7 \mathrm{fm}, A=8 \mathrm{fm}^{-1}$, and $B=-12.5 \mathrm{fm}^{-1}$ and they are given in Table 1.

The weight function $\rho(z)$ and another part of the upper radial wavefunction $F_{n \kappa}(z)$ which is $W(z)$ are found as follows by using (14) and (13), respectively:

$$
\begin{aligned}
& \rho(z)=z^{2 \mu}(1-z)^{2 \sqrt{\mu^{2}+\nu+\eta}}, \\
& W(z)=C_{n} z^{-2 \mu}(1-z)^{-2 \sqrt{\mu^{2}+\nu+\eta}} \\
& \cdot \frac{d^{n}}{d z^{n}}\left[z^{n+2 \mu}(1-z)^{n+2 \sqrt{\mu^{2}+\nu+\eta}}\right] \\
& \equiv P_{n}^{\left(2 \mu, 2 \sqrt{\mu^{2}+\nu+\eta}\right)}(1-2 z),
\end{aligned}
$$

where $P_{n}^{\left(2 \mu, 2 \sqrt{\left.\mu^{2}+v+\eta\right)}\right.}(1-2 z)$ is the jacobi polynomial. The upper radial wave function $F_{n \kappa}(z)$ is written as follows:

$$
\begin{aligned}
& F_{n \kappa}(z)=H(z) W(z) \\
& \quad=N_{n \kappa} z^{\mu}(1-z)^{\sqrt{\mu^{2}+\nu+\eta}} P_{n}^{\left(2 \mu, 2 \sqrt{\mu^{2}+\nu+\eta}\right)}(1-2 z),
\end{aligned}
$$

where $N_{n \kappa}$ is the normalization constant. The lower radial wave function $G_{n \kappa}(z)$ can be obtained by using (20).

\section{Pseudospin Symmetric Solution}

The sum of scalar potential with vector potential $\Sigma(r)$ and the difference between these potentials $\Delta(r)$ for the pseudospin symmetric case become as follows:

$$
\begin{aligned}
& \Sigma(r)=S_{p s}, \\
& \Delta(r)=\left[\frac{A e^{-2 b(r-L)}}{\left(q+p e^{-b(r-L)}\right)^{2}}+\frac{B e^{-b(r-L)}}{q+p e^{-b(r-L)}}\right],
\end{aligned}
$$

where $S_{p s}$ is a constant. By using (40), (24) reduces to following equation: 
TABLE 1: The bound state energy eigenvalues for the generalized Manning-Rosen potential in the spin symmetric case for $m=$ $4.76 \mathrm{fm}^{-1}$ and $S_{s}=3.9 \mathrm{fm}^{-1}$.

\begin{tabular}{ccccccccc}
\hline$l$ & $n$ & $\kappa<0$ & $(l, j)$ & $E_{n \kappa}\left(\mathrm{fm}^{-1}\right)$ & $n$ & $\kappa>0$ & $(l, j)$ & $E_{n \kappa}\left(\mathrm{fm}^{-1}\right)$ \\
\hline 1 & 0 & -2 & $0 p_{3 / 2}$ & 1.19206 & 0 & 1 & $0 p_{1 / 2}$ & 1.19206 \\
1 & 1 & -2 & $1 p_{3 / 2}$ & 2.67722 & 1 & 1 & $1 p_{1 / 2}$ & 2.67722 \\
1 & 2 & -2 & $2 p_{3 / 2}$ & 3.60544 & 2 & 1 & $2 p_{1 / 2}$ & 3.60544 \\
1 & 3 & -2 & $3 p_{3 / 2}$ & 4.20757 & 3 & 1 & $3 p_{1 / 2}$ & 4.20757 \\
2 & 0 & -3 & $0 d_{5 / 2}$ & 1.22410 & 0 & 2 & $0 d_{3 / 2}$ & 1.22410 \\
2 & 1 & -3 & $1 d_{5 / 2}$ & 2.69576 & 1 & 2 & $1 d_{3 / 2}$ & 2.69576 \\
2 & 2 & -3 & $2 d_{5 / 2}$ & 3.62088 & 2 & 2 & $2 d_{3 / 2}$ & 3.62088 \\
2 & 3 & -3 & $3 d_{5 / 2}$ & 4.22255 & 3 & 2 & $3 d_{3 / 2}$ & 4.22255 \\
3 & 0 & -4 & $0 f_{7 / 2}$ & 1.27064 & 0 & 3 & $0 f_{5 / 2}$ & 1.27064 \\
3 & 1 & -4 & $1 f_{7 / 2}$ & 2.72324 & 1 & 3 & $1 f_{5 / 2}$ & 2.72324 \\
3 & 2 & -4 & $2 f_{7 / 2}$ & 3.64383 & 2 & 3 & $2 f_{5 / 2}$ & 3.64383 \\
3 & 3 & -4 & $3 f_{7 / 2}$ & 4.24478 & 3 & 3 & $3 f_{5 / 2}$ & 4.24478 \\
\hline
\end{tabular}

$$
\begin{aligned}
& \left\{\frac{d^{2}}{d r^{2}}-\frac{\kappa(\kappa-1)}{r^{2}}-\left(E-m-S_{p s}\right)\right. \\
& \left.\cdot\left[\frac{A}{\left(p+q e^{b(r-L)}\right)^{2}}+\frac{B}{p+q e^{b(r-L)}}\right]\right\} G_{n \kappa}(r)+(E \\
& +m)\left(E-m-S_{p s}\right) G_{n \kappa}(r)=0 .
\end{aligned}
$$

Considering (27) instead of the centrifugal term and then changing variable like $z=p /\left(p+q e^{b(r-L)}\right)$, the following equation is found:

$$
\begin{aligned}
& \frac{d^{2} G_{n \kappa}(z)}{d z^{2}}+\frac{(1-2 z)}{z(1-z)} \frac{d G_{n \kappa}(z)}{d z} \\
& +\frac{\left(-\tilde{\mu}^{2}-\tilde{\nu} z-\tilde{\eta} z^{2}\right)}{z^{2}(1-z)^{2}} G_{n \kappa}(z)=0,
\end{aligned}
$$

where

$$
\begin{aligned}
\tilde{\mu}^{2} & =\frac{\kappa(\kappa-1) C_{0}-(E+m)\left(E-m-S_{p s}\right)}{b^{2}}, \\
\widetilde{v} & =\frac{\kappa(\kappa-1) C_{1}+\left(E-m-S_{p s}\right) B}{p b^{2}}, \\
\tilde{\eta} & =\frac{\kappa(\kappa-1) C_{2}+\left(E-m-S_{p s}\right) A}{p^{2} b^{2}} .
\end{aligned}
$$

Comparing (42) with (29), it is seen that replacing $\mu, \nu, \eta$, and $F_{n \kappa}(z)$ with $\tilde{\mu}, \tilde{v}, \tilde{\eta}$, and $G_{n \kappa}(z)$ in (29), respectively, (42) can be obtained. Therefore the bound state energy relation and the lower radial wave function for pseudospin symmetric limit are found writing $\widetilde{\mu}, \widetilde{\nu}, \widetilde{\eta}$, and $G_{n \kappa}(z)$ instead of $\mu, \nu, \eta$, and $F_{n \kappa}(z)$ in the solutions obtained in the spin symmetry limit. Then, the pseudospin symmetric bound state energy relation is directly written as follows:

$$
\begin{aligned}
& E^{2}-m^{2}-S_{p s}(E+m)-\kappa(\kappa-1) C_{0}-\frac{\kappa(\kappa-1) C_{1}+\left(E-m-S_{p s}\right) B}{2 p}-\frac{n(n+1) b^{2}}{2}-\frac{(2 n+1) b^{2}}{2} \\
& \cdot \sqrt{\frac{\kappa(\kappa-1) C_{0}-(E+m)\left(E-m-S_{p s}\right)}{b^{2}}-\frac{b^{2}}{2}\left(1+2 n+2 \sqrt{\left.\frac{\kappa(\kappa-1) C_{0}-(E+m)\left(E-m-S_{p s}\right)}{b^{2}}\right)}\right.} \\
& \cdot \sqrt{\frac{\kappa(\kappa-1)\left(C_{0} p^{2}+C_{1} p+C_{2}\right)+\left(E-m-S_{p s}\right)\left[A+B p-(E+m) p^{2}\right]}{b^{2} p^{2}}}=0 .
\end{aligned}
$$

The lower radial wave function is obtained in the following form:

$$
\begin{aligned}
& G_{n \kappa}(z) \\
& \quad=\widetilde{N}_{n \kappa} z^{\widetilde{\mu}}(1-z)^{\sqrt{\tilde{\mu}^{2}+\tilde{\nu}+\tilde{\eta}}} P_{n}^{\left(2 \widetilde{\mu}, 2 \sqrt{\tilde{\mu}^{2}+\tilde{\nu}+\tilde{\eta}}\right)}(1-2 z),
\end{aligned}
$$

where $\widetilde{N}_{n \kappa}$ is the normalization constant. The upper radial wave function $F_{n \kappa}(z)$ can be found by using (21).

From (44), bound state energy eigenvalues can be calculated for pseudospin symmetry case. We calculate some energy eigenvalues which are given in Table 2 for $q=0.8, p=$ $0.3, b=2 \mathrm{fm}^{-1}, L=7 \mathrm{fm}, A=-0.17 \mathrm{fm}^{-1}$, and $B=-0.9 \mathrm{fm}^{-1}$ depending on quantum numbers $n, \kappa$.

\section{Effects of Parameters on Energy Eigenvalues}

In this section, we investigate dependence of energy eigenvalues to parameters graphically for $2 p_{3 / 2}, 2 d_{5 / 2}$, and $2 f_{7 / 2}$ states in the spin symmetric limit and for $2 s_{1 / 2}, 2 p_{3 / 2}$, and $2 d_{5 / 2}$ states in the pseudospin symmetric limit.

The effects of $b, p, L, q, m, S_{s}$, and $S_{p s}$ parameters on energy eigenvalues is given in Figures $2,3,4,5,6$, and 7 , respectively. It is seen from these figures that the energy eigenvalues increase as $b, m$, and $S_{s}$ increase and decrease as $p, L$, and $q$ increase in the spin symmetric case. On the other hand in the pseudospin symmetric case, energy eigenvalues increase as $q, m$, and $S_{p s}$ increase and decrease as $b, p$, and $L$ increase. Decrease of the energy eigenvalues means that the bound states become less bounded and increase in energy 
TABLE 2: The bound state energy eigenvalues for the generalized Manning-Rosen potential in the pseudospin symmetric case for $m=$ $4.76 \mathrm{fm}^{-1}$ and $S_{p s}=-0.1 \mathrm{fm}^{-1}$.

\begin{tabular}{ccccccccc}
\hline$\tilde{l}$ & $n$ & $\kappa<0$ & $(l, j)$ & $\begin{array}{c}E_{n \kappa} \\
\left(\mathrm{fm}^{-1}\right)\end{array}$ & $n-1$ & $\kappa>0$ & $(l+2, j+1)$ & $\begin{array}{c}E_{n \kappa} \\
\left(\mathrm{fm}^{-1}\right)\end{array}$ \\
\hline 1 & 1 & -1 & $1 s_{1 / 2}$ & -4.73362 & 0 & 2 & $0 d_{3 / 2}$ & -4.73362 \\
1 & 2 & -1 & $2 s_{1 / 2}$ & -4.19017 & 1 & 2 & $1 d_{3 / 2}$ & -4.19017 \\
1 & 3 & -1 & $3 s_{1 / 2}$ & -2.89434 & 2 & 2 & $2 d_{3 / 2}$ & -2.89434 \\
2 & 1 & -2 & $1 p_{3 / 2}$ & -4.74120 & 0 & 3 & $0 f_{5 / 2}$ & -4.74120 \\
2 & 2 & -2 & $2 p_{3 / 2}$ & -4.20134 & 1 & 3 & $1 f_{5 / 2}$ & -4.20134 \\
2 & 3 & -2 & $3 p_{3 / 2}$ & -2.91352 & 2 & 3 & $2 f_{5 / 2}$ & -2.91352 \\
3 & 1 & -3 & $1 d_{5 / 2}$ & -4.75252 & 0 & 4 & $0 g_{7 / 2}$ & -4.75252 \\
3 & 2 & -3 & $2 d_{5 / 2}$ & -4.21801 & 1 & 4 & $1 g_{7 / 2}$ & -4.21801 \\
3 & 3 & -3 & $3 d_{5 / 2}$ & -2.94202 & 2 & 4 & $2 g_{7 / 2}$ & -2.94202 \\
\hline
\end{tabular}

eigenvalues indicates that the bound states become more tightly bounded.

In Figure 4(a), the bound states do not occur in $2 d_{5 / 2}$ and $2 f_{7 / 2}$ for $L=1$ because the energy values of $2 d_{5 / 2}$ and $2 f_{7 / 2}$ are greater than the mass of nucleon. In order to obtain a bound state the binding energies of nuclear states must be smaller than the mass of nucleon $\left(m \approx 4.76 \mathrm{fm}^{-1} \approx\right.$ $939 \mathrm{MeV}$ ). Moreover, it is seen from Figure 4 that the binding energies of nuclear states are getting closer to each other as $L$ increases.

\section{Special Cases}

In certain limits and depending on values of the potential parameters, the generalized Manning-Rosen potential can be reduced to various potentials that are used to explain different physical processes. The bound states energy eigenvalues for these potentials are investigated and compared with the previous studies.

7.1. Manning-Rosen Potential. If parameters of the generalized Manning-Rosen potential are chosen as $p=-1, b=1 / \beta$, $q=e^{b L}, A=\alpha(\alpha-1) / 2 m \beta^{2}$, and $B=-\widetilde{A} / 2 m \beta^{2}$, the following potential is found:

$$
V(r)=\frac{1}{2 m \beta^{2}}\left[\frac{\alpha(\alpha-1) e^{-2 r / \beta}}{\left(1-e^{-r / \beta}\right)^{2}}-\frac{\widetilde{A} e^{-r / \beta}}{1-e^{-r / \beta}}\right] .
$$

This potential is known as the Manning-Rosen potential [68]. From (44) and (37), the bound energy eigenvalues of the Manning-Rosen potential can be obtained for pseudospin symmetric and spin symmetric cases. For $L=17.5, p=$ $-1, m=1, S_{p s}=-6, S_{s}=6, \alpha=1.5, \beta=20$, and $\widetilde{A}=30.52$, the bound energy eigenvalues for pseudospin and spin symmetric cases are calculated and these are given in Tables 3 and 4 , respectively. In Table 3 our results are compared with those presented in [27]. Also if $L=0.05$ and the values of parameters which are given in [26] are used for spin symmetric case, it can be seen that our results are consistent with the results of [26].
7.2. Kratzer-Fues Potential. By taking $p=-1, q=e^{b L}$, $A=D_{e} r_{e}^{2} b^{2}, B=-2 D_{e} r_{e} b$, and $\lim _{b \rightarrow 0}$ in (1), the generalized Manning-Rosen potential reduces to Kratzer-Fues potential $[80,81]$ :

$$
\begin{aligned}
V_{\mathrm{KF}}(r) & =\lim _{b \rightarrow 0}\left[\frac{D_{e} r_{e}^{2} b^{2} e^{-2 b r}}{\left(1-e^{-b r}\right)^{2}}-\frac{2 D_{e} r_{e} b e^{-b r}}{1-e^{-b r}}\right] \\
& =-D_{e}\left(\frac{2 r_{e}}{r}-\frac{r_{e}^{2}}{r^{2}}\right),
\end{aligned}
$$

where $D_{e}$ and $r_{e}$ are the dissociation energy and equilibrium internuclear length, respectively.

For $L=4.9, p=-1, m=5 \mathrm{fm}^{-1}, S_{p s}=0, S_{s}=0$, $b=0.000002, D_{e}=1.25 \mathrm{fm}^{-1}$, and $r_{e}=0.35 \mathrm{fm}$, the bound state energy eigenvalues for Kratzer-Fues potential are found in the pseudospin and spin symmetric cases by using (44) and (37), respectively, and these are given in Tables 5 and 6. it can be seen from these tables that our energy eigenvalues are consistent with those obtained in [46] both for pseudospin and for spin symmetric cases.

7.3. Morse Potential. Considering $q=1, A=D_{e}, B=-2 D_{e}$, $b=a$, and $L=r_{e}$ and taking $\lim _{p \rightarrow 0}$, the generalized Manning-Rosen potential reduces to the Morse potential [74]:

$$
\begin{aligned}
V_{\mathrm{M}}(r) & =\lim _{p \rightarrow 0}\left[\frac{D_{e} e^{-2 a\left(r-r_{e}\right)}}{\left(1+p e^{-a\left(r-r_{e}\right)}\right)^{2}}-\frac{2 D_{e} e^{-a\left(r-r_{e}\right)}}{1+p e^{-a\left(r-r_{e}\right)}}\right] \\
& \approx D_{e}\left[e^{-2 a\left(r-r_{e}\right)}-2 e^{-a\left(r-r_{e}\right)}\right]
\end{aligned}
$$

where $r_{e}$ is the equilibrium distance and $a$ is related to the range of the potential well. We can not take $p=0$ because the solutions of (44) and (37) go to infinity.

The bound state energy eigenvalues for the Morse potential in the pseudospin and spin symmetric cases are given in Tables 7 and 8, respectively, for $q=1, m=10 \mathrm{fm}^{-1}$, $S_{p s}=-10 \mathrm{fm}^{-1}, S_{s}=10 \mathrm{fm}^{-1}, b=0.988879, D_{e}=5 \mathrm{fm}^{-1}$, $L=2.40873$, and $p=0.000000000013$. Equations (44) and (37) are used to calculate the bound state energy eigenvalues. These tables show that the energy eigenvalues obtained for pseudospin and spin symmetric cases are compatible with the energy eigenvalues of $[49,50]$ when they are compared.

If the parameters are chosen as $q=e^{b L}, A=0, B=-V_{0}$, and $\lim _{p \rightarrow 0}$ in (1), the generalized Manning-Rosen potential reduces to the cusp potential [90]. The energy eigenvalues for this potential also can be calculated by using (44) in the pseudospin symmetry limit and (37) in the spin symmetry limit.

7.4. Hulthen Potential. Hulthen potential [84] can be obtained as follows by taking $p=-1, q=e^{b L}, b=2 \alpha, A=0$, and $B=-V_{0}$ in (1):

$$
V_{\mathrm{H}}(r)=-V_{0} \frac{e^{-2 \alpha r}}{1-e^{-2 \alpha r}},
$$




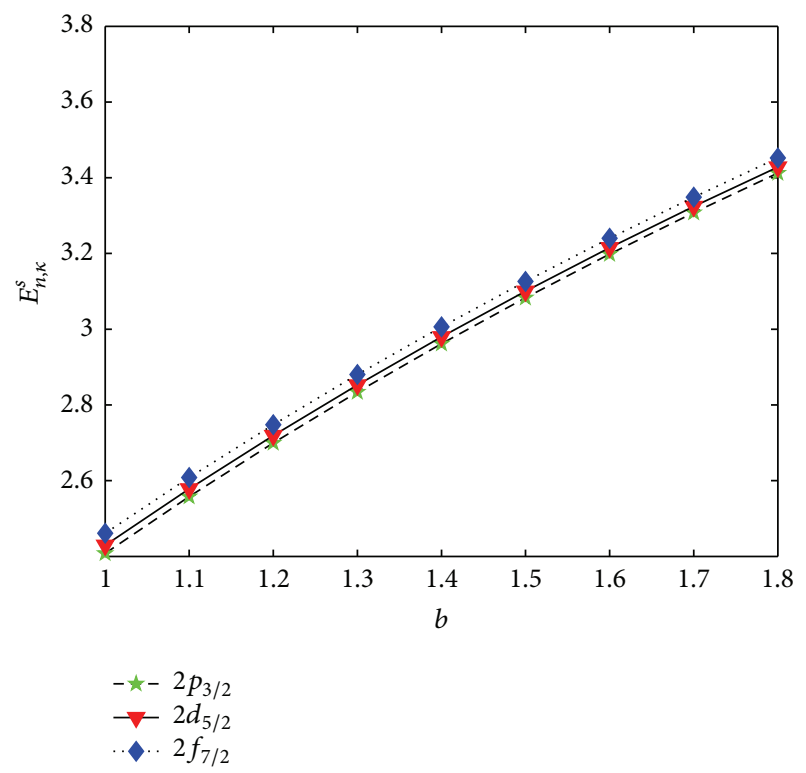

(a)

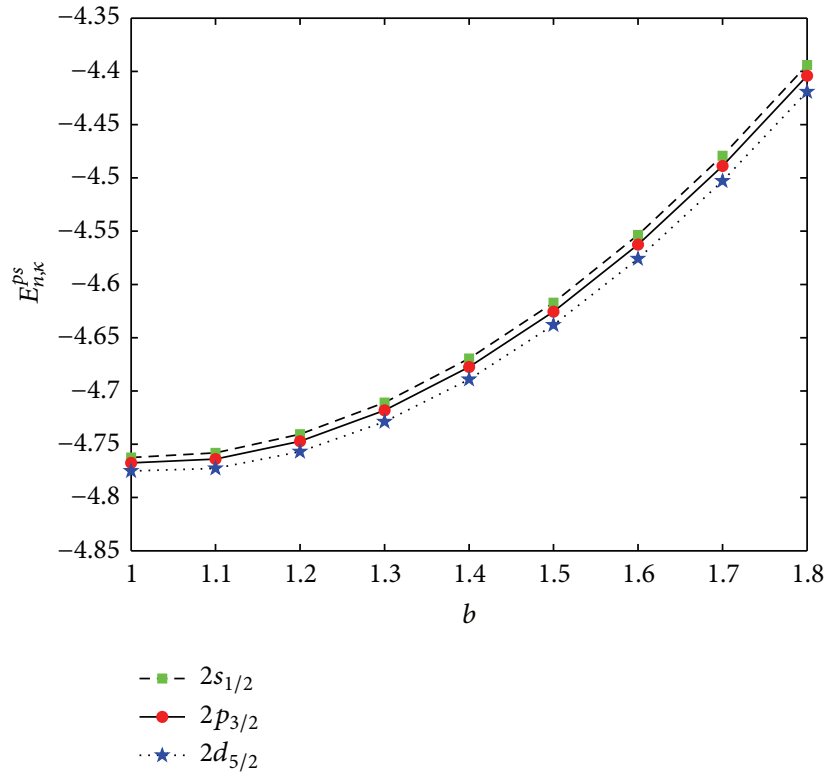

(b)

Figure 2: (a) Energy eigenvalues versus $b$ in spin symmetric limit for $A=8 \mathrm{fm}^{-1}, B=-12.5 \mathrm{fm}^{-1}, S_{s}=3.9 \mathrm{fm}^{-1}, L=7 \mathrm{fm}, m=4.76 \mathrm{fm}{ }^{-1}$, $q=0.8$, and $p=0.65$. (b) Energy eigenvalues versus $b$ in pseudospin symmetric limit for $A=-0.17 \mathrm{fm}^{-1}, B=-0.9 \mathrm{fm}^{-1}, S_{p s}=-0.1 \mathrm{fm}^{-1}$, $L=7 \mathrm{fm}, m=4.76 \mathrm{fm}^{-1}, q=0.8$, and $p=0.3$.

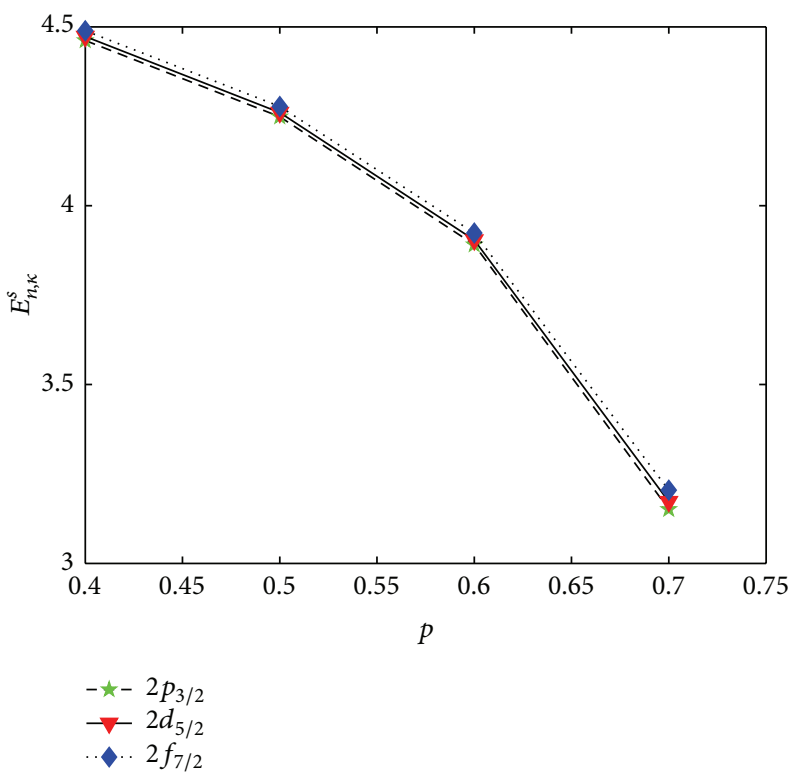

(a)

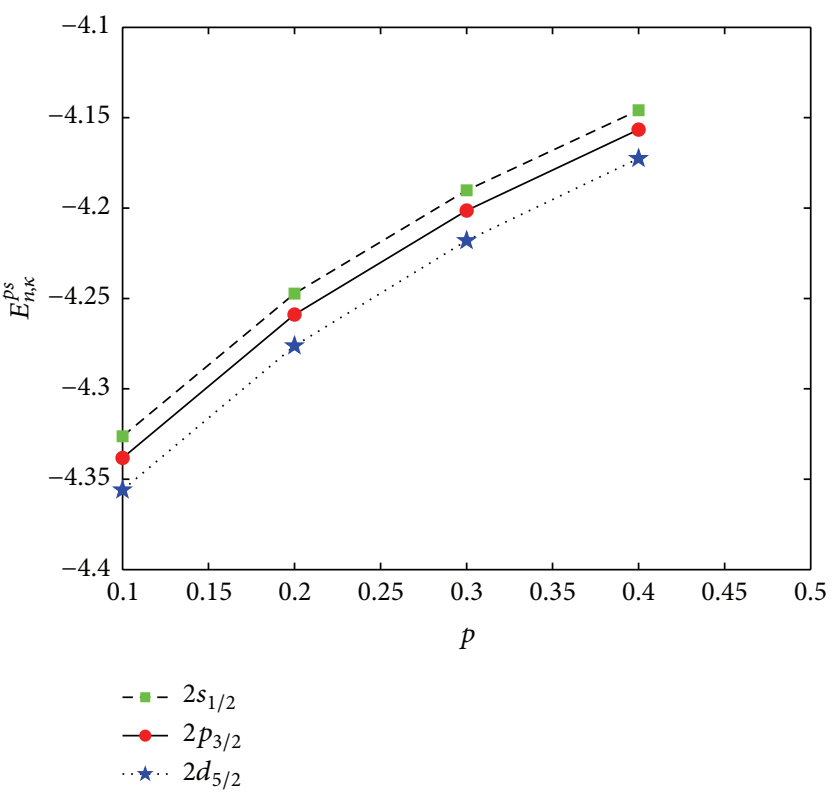

(b)

FIGURE 3: (a) Energy eigenvalues versus $p$ in spin symmetric limit for $A=8 \mathrm{fm}^{-1}, B=-12.5 \mathrm{fm}^{-1}, S_{s}=3.9 \mathrm{fm}^{-1}, L=7 \mathrm{fm}, m=4.76 \mathrm{fm}{ }^{-1}$, $b=2 \mathrm{fm}^{-1}$, and $q=0.8$. (b) Energy eigenvalues versus $p$ in pseudospin symmetric limit for $A=-0.17 \mathrm{fm}^{-1}, B=-0.9 \mathrm{fm}^{-1}, S_{p s}=-0.1 \mathrm{fm}^{-1}$, $L=7 \mathrm{fm}, m=4.76 \mathrm{fm}^{-1}, b=2 \mathrm{fm}^{-1}$, and $q=0.8$.

where $V_{0}$ is the dept of the potential and $\alpha$ is the screening parameter of the potential.

By using (44) and (37), bound state energy eigenvalues are calculated for Hulthen potential in the pseudospin and spin symmetric cases for $b=0.1 \mathrm{fm}, L=15, B=-4 \mathrm{fm}^{-1}$,
$S_{p s}=-5 \mathrm{fm}^{-1}, S_{s}=5 \mathrm{fm}^{-1}$, and $m=5 \mathrm{fm}^{-1}$. These energy eigenvalues are compared with the energy eigenvalues of [40] in Tables 9 and 10. Here our energy eigenvalues are little different from results of [40]. The reason of this is approximation that is applied to the centrifugal term. The authors of [40] 


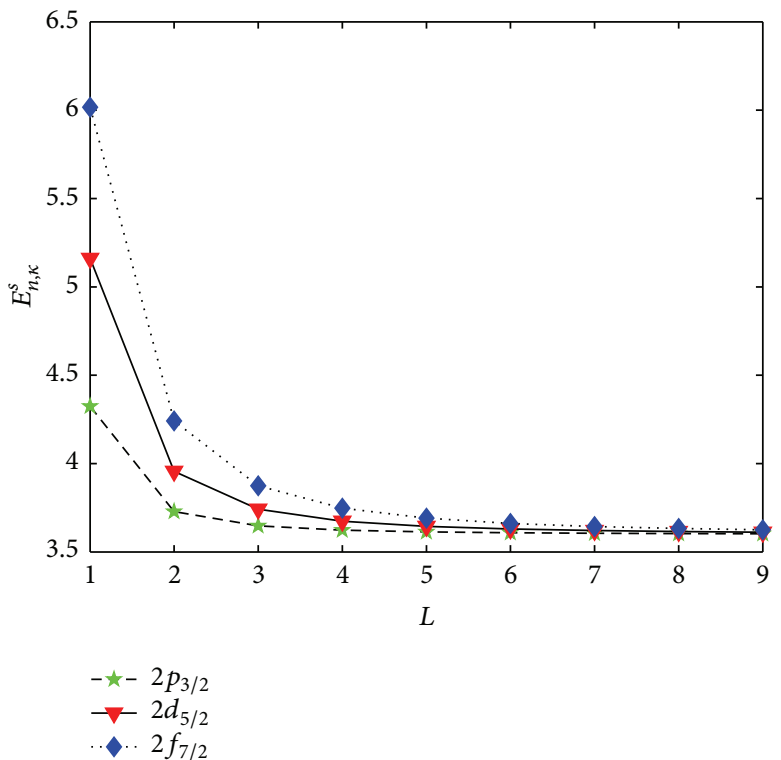

(a)

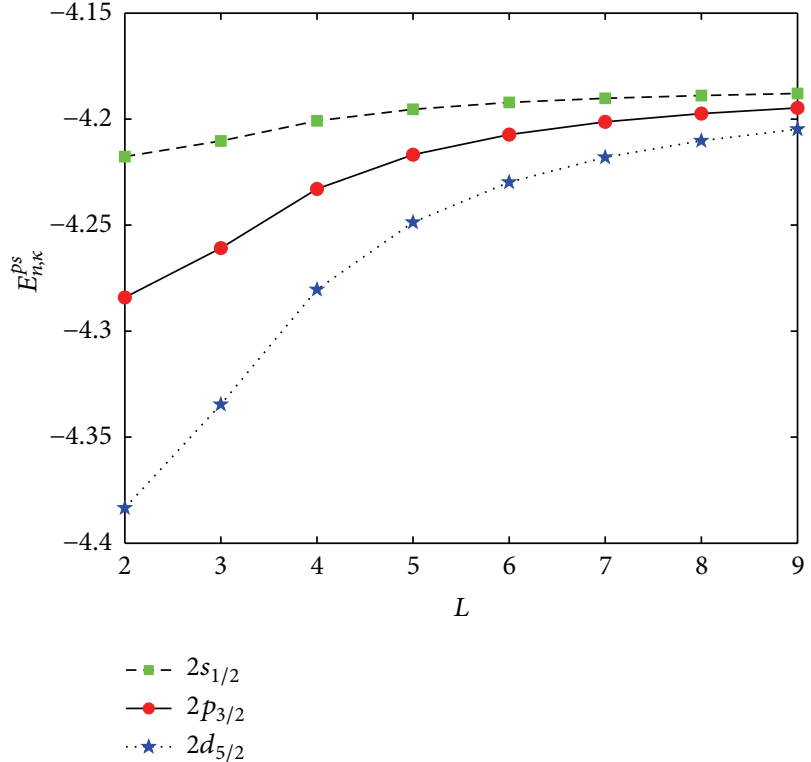

(b)

Figure 4: (a) Energy eigenvalues versus $L$ in spin symmetric limit for $A=8 \mathrm{fm}^{-1}, B=-12.5 \mathrm{fm}^{-1}, S_{s}=3.9 \mathrm{fm}^{-1}, m=4.76 \mathrm{fm}^{-1}, b=2 \mathrm{fm}^{-1}$, $p=0.65$, and $q=0.8$. (b) Energy eigenvalues versus $L$ in pseudospin symmetric limit for $A=-0.17 \mathrm{fm}^{-1}, B=-0.9 \mathrm{fm}^{-1}, S_{p s}=-0.1 \mathrm{fm}^{-1}$, $m=4.76 \mathrm{fm}^{-1}, b=2 \mathrm{fm}^{-1}, p=0.3$, and $q=0.8$.

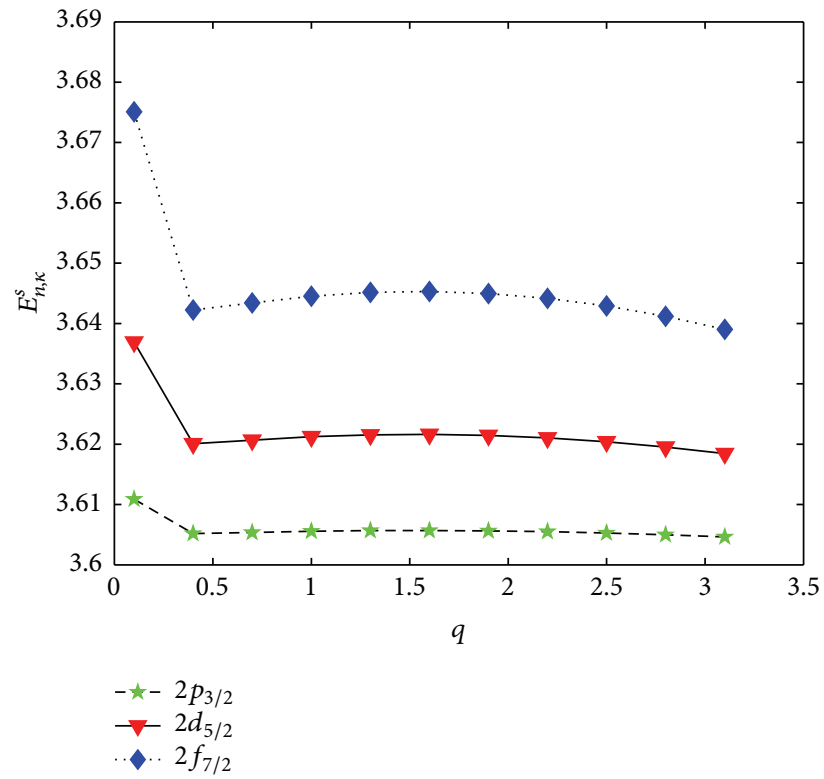

(a)

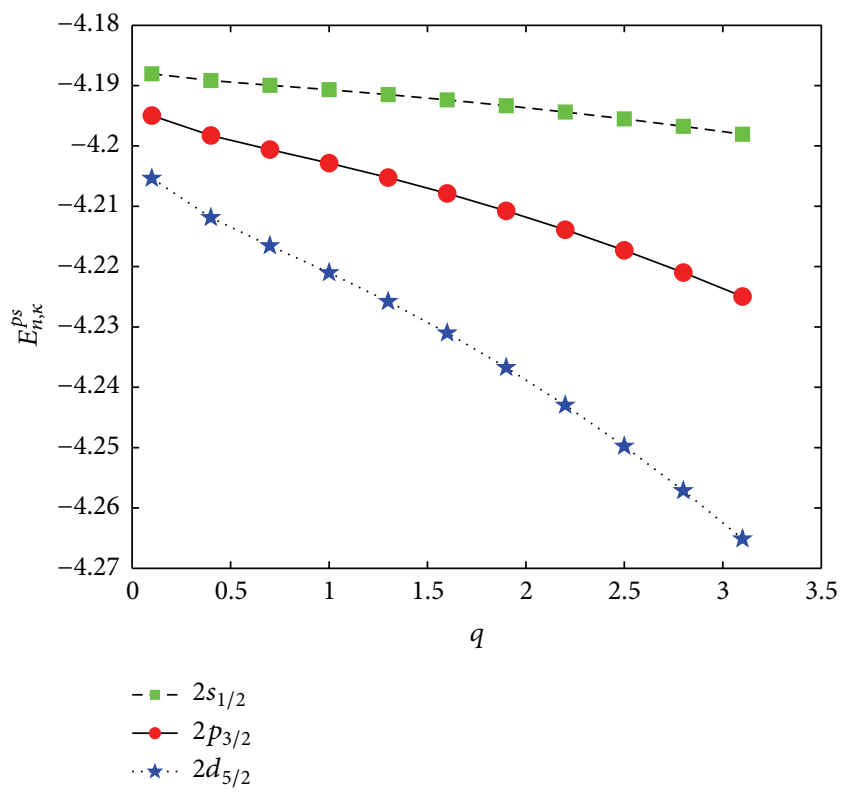

(b)

Figure 5: (a) Energy eigenvalues versus $q$ in spin symmetric limit for $A=8 \mathrm{fm}^{-1}, B=-12.5 \mathrm{fm}^{-1}, S_{s}=3.9 \mathrm{fm}^{-1}, m=4.76 \mathrm{fm}{ }^{-1}, L=7 \mathrm{fm}$, $b=2 \mathrm{fm}^{-1}$, and $p=0.65$. (b) Energy eigenvalues versus $q$ in pseudospin symmetric limit for $A=-0.17 \mathrm{fm}^{-1}, B=-0.9 \mathrm{fm}^{-1}, S_{p s}=-0.1 \mathrm{fm}^{-1}$, $m=4.76 \mathrm{fm}^{-1}, L=7 \mathrm{fm}, b=2 \mathrm{fm}^{-1}$, and $p=0.3$.

have used the Greene-Aldrich [91] approximation but we use the Pekeris [89] approximation instead of centrifugal term.

7.5. Yukawa Potential. Yukawa potential [85] can be obtained by considering $q=e^{b L}, b=2 \alpha, B=-2 \alpha V_{0}, A=0$, and $p=-1$ in (1):

$$
V_{\mathrm{Y}}(r)=-V_{0} e^{-\alpha r}\left(\frac{2 \alpha e^{-\alpha r}}{1-e^{-2 \alpha r}}\right) \approx-V_{0} \frac{e^{-\alpha r}}{r},
$$

where $1 / r \approx\left(2 \alpha e^{-\alpha r} /\left(1-e^{-2 \alpha r}\right)\right)$ and $\alpha$ is the range of the nucleon force in the meson theory $[55,85]$. 


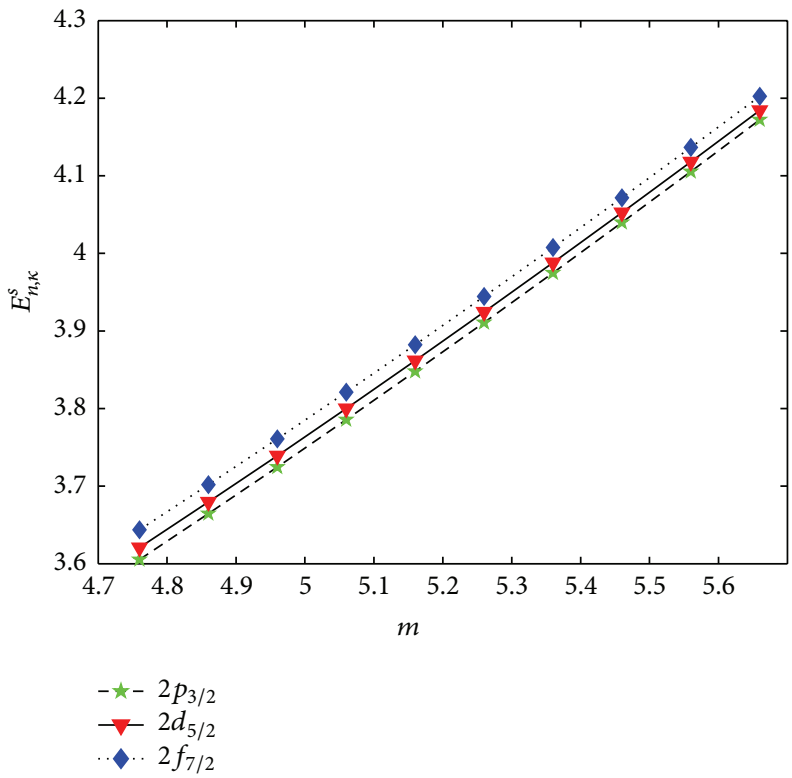

(a)

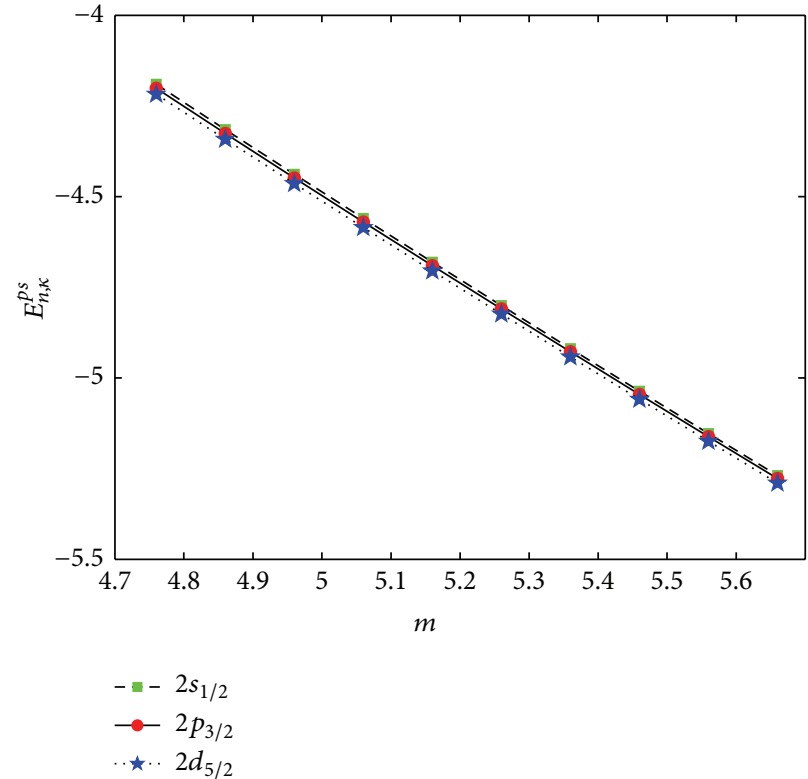

(b)

FIgURE 6: (a) Energy eigenvalues versus $m$ in spin symmetric limit for $A=8 \mathrm{fm}^{-1}, B=-12.5 \mathrm{fm}^{-1}, S_{s}=3.9 \mathrm{fm}^{-1}, L=7 \mathrm{fm}, b=2 \mathrm{fm}^{-1}$, $q=0.8$, and $p=0.65$. (b) Energy eigenvalues versus $m$ in pseudospin symmetric limit for $A=-0.17 \mathrm{fm}^{-1}, B=-0.9 \mathrm{fm}^{-1}, S_{p s}=-0.1 \mathrm{fm}^{-1}$, $L=7 \mathrm{fm}, b=2 \mathrm{fm}^{-1}, q=0.8$, and $p=0.3$.

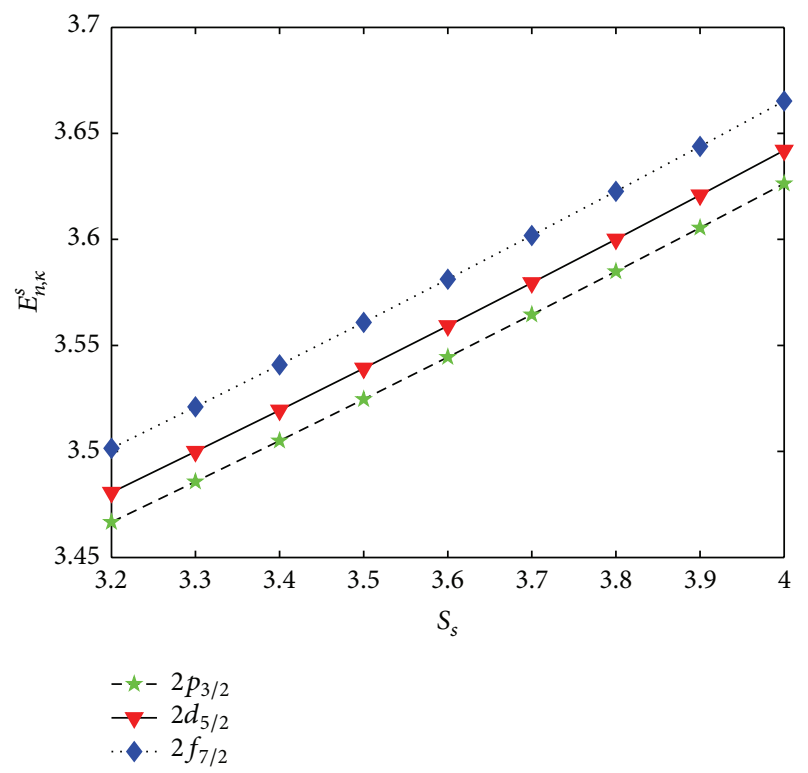

(a)

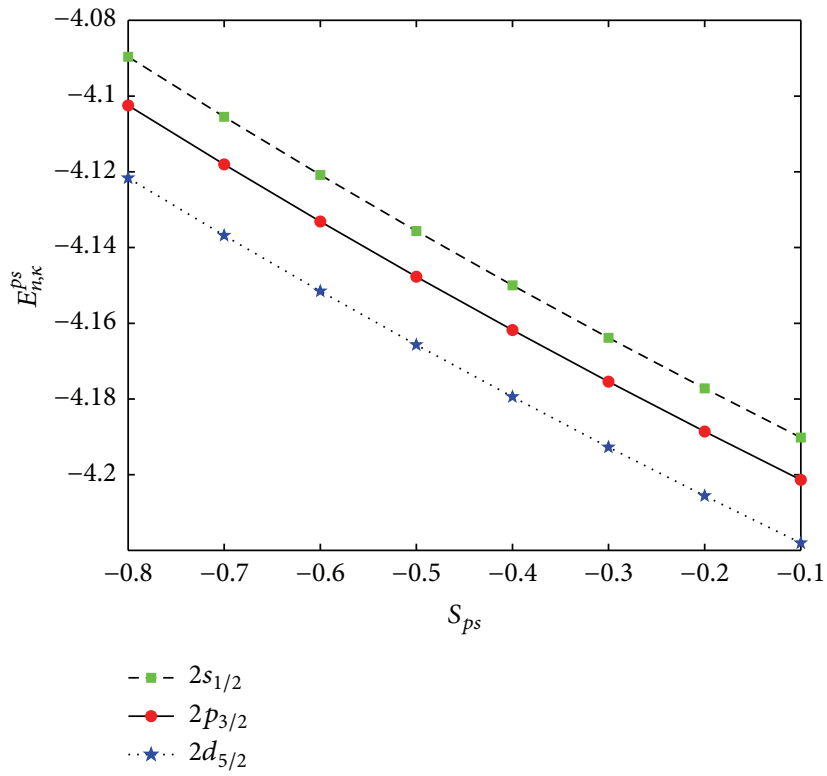

(b)

Figure 7: (a) Energy eigenvalues versus $S_{s}$ in spin symmetric limit for $A=8 \mathrm{fm}^{-1}, B=-12.5 \mathrm{fm}^{-1}, m=4.76 \mathrm{fm}^{-1}, L=7 \mathrm{fm}, b=2 \mathrm{fm}^{-1}$, $q=0.8$, and $p=0.65$. (b) Energy eigenvalues versus $S_{p s}$ in pseudospin symmetric limit for $A=-0.17 \mathrm{fm}^{-1}, B=-0.9 \mathrm{fm}^{-1}, m=4.76 \mathrm{fm}^{-1}$, $L=7 \mathrm{fm}, b=2 \mathrm{fm}^{-1}, q=0.8$, and $p=0.3$.

The energy eigenvalues for this potential are found in the pseudospin and spin symmetric cases by using (44) and (37), respectively, for $b=0.2 \mathrm{fm}, L=0.0001, V_{0}=1 \mathrm{fm}^{-1}$, $S_{p s}=-5 \mathrm{fm}^{-1}, S_{s}=5 \mathrm{fm}^{-1}$, and $m=5 \mathrm{fm}^{-1}$. These energy eigenvalues for pseudospin symmetry are compared with the results of $[55,56]$ in Table 11 and spin symmetric energy eigenvalues are compared with the results of [56] in Table 12. As shown in Tables 11 and 12, pseudospin and spin doublets have not occurred in results of $[55,56]$. Besides, in $1 s_{1 / 2}$ and $2 p_{3 / 2}$ for pseudospin symmetry and in $1 p_{3 / 2}$ for spin 
TABLE 3: The bound state energy eigenvalues for the Manning-Rosen potential in the pseudospin symmetric case for $m=1$ and $S_{p s}=-6$. Here first states column is according to $(l, j)$ and second states column is according to $(l+2, j+1)$.

\begin{tabular}{|c|c|c|c|c|c|c|c|}
\hline$|n, \widetilde{l}, \kappa\rangle$ & State & $E_{n \kappa}$ & $E_{n \kappa}[27]$ & $|n-1, \widetilde{l}, \kappa\rangle$ & State & $E_{n \kappa}$ & $E_{n \kappa}[27]$ \\
\hline$|1,1,-1\rangle$ & $1 s_{1 / 2}$ & -4.99867 & -4.99866 & $|0,1,2\rangle$ & $0 d_{3 / 2}$ & -4.99867 & -4.99866 \\
\hline$|1,2,-2\rangle$ & $1 p_{3 / 2}$ & -4.99774 & -4.99772 & $|0,2,3\rangle$ & $0 f_{5 / 2}$ & -4.99774 & -4.99772 \\
\hline$|1,3,-3\rangle$ & $1 d_{5 / 2}$ & -4.99657 & -4.99656 & $|0,3,4\rangle$ & $0 g_{7 / 2}$ & -4.99657 & -4.99656 \\
\hline$|1,4,-4\rangle$ & $1 f_{7 / 2}$ & -4.99517 & -4.99517 & $|0,4,5\rangle$ & $0 h_{9 / 2}$ & -4.99517 & -4.99517 \\
\hline
\end{tabular}

TABLE 4: The bound state energy eigenvalues for the Manning-Rosen potential in the spin symmetric case for $m=1$ and $S_{s}=6$. Here first and second states columns are according to $(l, j)$.

\begin{tabular}{llclrr}
\hline$|n, l, \kappa\rangle$ & State & $E_{n \kappa}$ & $|n, l, \kappa\rangle$ & State & $E_{n \kappa}$ \\
\hline$|0,1,-2\rangle$ & $0 p_{3 / 2}$ & 4.99945 & $|0,1,1\rangle$ & $0 p_{1 / 2}$ & 4.99945 \\
$|0,2,-3\rangle$ & $0 d_{5 / 2}$ & 4.99882 & $|0,2,2\rangle$ & $0 d_{3 / 2}$ & 4.99882 \\
$|0,3,-4\rangle$ & $0 f_{7 / 2}$ & 4.99795 & $|0,3,3\rangle$ & $0 f_{5 / 2}$ & 4.99795 \\
$|0,4,-5\rangle$ & $0 g_{9 / 2}$ & 4.99685 & $|0,4,4\rangle$ & $0 g_{7 / 2}$ & 4.99685 \\
\hline
\end{tabular}

TABLE 5: The bound state energy eigenvalues for the Kratzer-Fues potential in the pseudospin symmetric case for $m=5 \mathrm{fm}^{-1}$ and $S_{p s}=0 \mathrm{fm}{ }^{-1}$. Here first states column is according to $(l, j)$ and second states column is according to $(l+2, j+1)$.

\begin{tabular}{|c|c|c|c|c|c|c|c|}
\hline$|n, \tilde{l}, \kappa\rangle$ & State & $E_{n \kappa}$ & $E_{n \kappa}[46]$ & $|n-1, \widetilde{l}, \kappa\rangle$ & State & $E_{n \kappa}$ & $E_{n \kappa}[46]$ \\
\hline$|1,1,-1\rangle$ & $1 s_{1 / 2}$ & -4.671859 & -4.672305 & $|0,1,2\rangle$ & $0 d_{3 / 2}$ & -4.671859 & -4.672305 \\
\hline$|1,2,-2\rangle$ & $1 p_{3 / 2}$ & -4.860367 & -4.860421 & $|0,2,3\rangle$ & $0 f_{5 / 2}$ & -4.860367 & -4.860421 \\
\hline$|1,3,-3\rangle$ & $1 d_{5 / 2}$ & -4.916765 & -4.916782 & $|0,3,4\rangle$ & $0 g_{7 / 2}$ & -4.916765 & -4.916782 \\
\hline$|1,4,-4\rangle$ & $1 f_{7 / 2}$ & -4.943945 & -4.943953 & $|0,4,5\rangle$ & $0 h_{9 / 2}$ & -4.943945 & -4.943953 \\
\hline$|2,1,-1\rangle$ & $2 s_{1 / 2}$ & -4.833423 & -4.833547 & $|1,1,2\rangle$ & $1 d_{3 / 2}$ & -4.833423 & -4.833547 \\
\hline$|2,2,-2\rangle$ & $2 p_{3 / 2}$ & -4.913175 & -4.913195 & $|1,2,3\rangle$ & $1 f_{5 / 2}$ & -4.913175 & -4.913195 \\
\hline$|2,3,-3\rangle$ & $2 d_{5 / 2}$ & -4.942933 & -4.942941 & $|1,3,4\rangle$ & $1 g_{7 / 2}$ & -4.942933 & -4.942941 \\
\hline$|2,4,-4\rangle$ & $2 f_{7 / 2}$ & -4.959099 & -4.959104 & $|1,4,5\rangle$ & $1 h_{9 / 2}$ & -4.959099 & -4.959104 \\
\hline
\end{tabular}

TABLE 6: The bound state energy eigenvalues for the Kratzer-Fues potential in the spin symmetric case for $m=5 \mathrm{fm}^{-1}$ and $S_{s}=0 \mathrm{fm}^{-1}$. Here first and second states columns are according to $(l, j)$.

\begin{tabular}{|c|c|c|c|c|c|c|c|}
\hline$|n, l, \kappa\rangle$ & State & $E_{n \kappa}$ & $E_{n \kappa}[46]$ & $|n, l, \kappa\rangle$ & State & $E_{n \kappa}$ & $E_{n \kappa}[46]$ \\
\hline$|0,1,-2\rangle$ & $0 p_{3 / 2}$ & 4.686684 & 4.686570 & $|0,1,1\rangle$ & $0 p_{1 / 2}$ & 4.686684 & 4.686570 \\
\hline$|0,2,-3\rangle$ & $0 d_{5 / 2}$ & 4.825721 & 4.825688 & $|0,2,2\rangle$ & $0 d_{3 / 2}$ & 4.825721 & 4.825688 \\
\hline$|0,3,-4\rangle$ & $0 f_{7 / 2}$ & 4.893179 & 4.893167 & $|0,3,3\rangle$ & $0 f_{5 / 2}$ & 4.893179 & 4.893167 \\
\hline$|0,4,-5\rangle$ & $0 g_{9 / 2}$ & 4.928792 & 4.928786 & $|0,4,4\rangle$ & $0 g_{7 / 2}$ & 4.928792 & 4.928786 \\
\hline$|1,1,-2\rangle$ & $1 p_{3 / 2}$ & 4.840692 & 4.840668 & $|1,1,1\rangle$ & $1 p_{1 / 2}$ & 4.840692 & 4.840668 \\
\hline$|1,2,-3\rangle$ & $1 d_{5 / 2}$ & 4.896919 & 4.896810 & $|1,2,2\rangle$ & $1 d_{3 / 2}$ & 4.896919 & 4.896810 \\
\hline$|1,3,-4\rangle$ & $1 f_{7 / 2}$ & 4.930006 & 4.930002 & $|1,3,3\rangle$ & $1 f_{5 / 2}$ & 4.930006 & 4.930002 \\
\hline$|1,4,-5\rangle$ & $1 g_{9 / 2}$ & 4.949914 & 4.949911 & $|1,4,4\rangle$ & $1 g_{7 / 2}$ & 4.949914 & 4.949911 \\
\hline
\end{tabular}

TABLE 7: The bound state energy eigenvalues for the Morse potential in the pseudospin symmetric case for $m=10 \mathrm{fm}^{-1}$ and $S_{p s}=-10 \mathrm{fm}^{-1}$. Here first states column is according to $(l, j)$ and second states column is according to $(l+2, j+1)$.

\begin{tabular}{|c|c|c|c|c|c|c|c|}
\hline$|n, \widetilde{l}, \kappa\rangle$ & State & $E_{n \kappa}$ & $E_{n \kappa}[49,50]$ & $|n-1, \widetilde{l}, \kappa\rangle$ & State & $E_{n \kappa}$ & $E_{n \kappa}[49,50]$ \\
\hline$|1,1,-1\rangle$ & $1 s_{1 / 2}$ & -0.0064125 & -0.0064123 & $|0,1,2\rangle$ & $0 d_{3 / 2}$ & -0.0064125 & -0.0064123 \\
\hline$|1,2,-2\rangle$ & $1 p_{3 / 2}$ & -0.0155780 & -0.0155771 & $|0,2,3\rangle$ & $0 f_{5 / 2}$ & -0.0155780 & -0.0155771 \\
\hline$|1,3,-3\rangle$ & $1 d_{5 / 2}$ & -0.0243675 & -0.0243659 & $|0,3,4\rangle$ & $0 g_{7 / 2}$ & -0.0243675 & -0.0243659 \\
\hline$|1,4,-4\rangle$ & $1 f_{7 / 2}$ & -0.0305324 & -0.0305297 & $|0,4,5\rangle$ & $0 h_{9 / 2}$ & -0.0305324 & -0.0305297 \\
\hline$|2,1,-1\rangle$ & $2 s_{1 / 2}$ & -0.0070207 & -0.0070204 & $|1,1,2\rangle$ & $1 d_{3 / 2}$ & -0.0070207 & -0.0070204 \\
\hline$|2,2,-2\rangle$ & $2 p_{3 / 2}$ & -0.0190450 & -0.0190441 & $|1,2,3\rangle$ & $1 f_{5 / 2}$ & -0.0190450 & -0.0190441 \\
\hline$|2,3,-3\rangle$ & $2 d_{5 / 2}$ & -0.0337736 & -0.0337719 & $|1,3,4\rangle$ & $1 g_{7 / 2}$ & -0.0337736 & -0.0337719 \\
\hline$|2,4,-4\rangle$ & $2 f_{7 / 2}$ & -0.0492178 & -0.0492150 & $|1,4,5\rangle$ & $1 h_{9 / 2}$ & -0.0492178 & -0.0492150 \\
\hline
\end{tabular}


TABLE 8: The bound state energy eigenvalues for the Morse potential in the spin symmetric case for $m=10 \mathrm{fm}^{-1}$ and $S_{s}=10 \mathrm{fm}^{-1}$. Here first and second states columns are according to $(l, j)$.

\begin{tabular}{|c|c|c|c|c|c|c|c|}
\hline$|n, l, \kappa\rangle$ & State & $E_{n \kappa}$ & $E_{n \kappa}[49]$ & $|n, l, \kappa\rangle$ & State & $E_{n \kappa}$ & $E_{n \kappa}[49]$ \\
\hline$|0,1,-2\rangle$ & $0 p_{3 / 2}$ & 0.0188480 & 0.0188481 & $|0,1,1\rangle$ & $0 p_{1 / 2}$ & 0.0188480 & 0.0188481 \\
\hline$|0,2,-3\rangle$ & $0 d_{5 / 2}$ & 0.0336560 & 0.0336562 & $|0,2,2\rangle$ & $0 d_{3 / 2}$ & 0.0336560 & 0.0336562 \\
\hline$|0,3,-4\rangle$ & $0 f_{7 / 2}$ & 0.0525267 & 0.0525273 & $|0,3,3\rangle$ & $0 f_{5 / 2}$ & 0.0525267 & 0.0525273 \\
\hline$|0,4,-5\rangle$ & $0 g_{9 / 2}$ & 0.0754340 & 0.0754350 & $|0,4,4\rangle$ & $0 g_{7 / 2}$ & 0.0754340 & 0.0754350 \\
\hline$|1,1,-2\rangle$ & $1 p_{3 / 2}$ & 0.0899995 & 0.0899995 & $|1,1,1\rangle$ & $1 p_{1 / 2}$ & 0.0899995 & 0.0899995 \\
\hline$|1,2,-3\rangle$ & $1 d_{5 / 2}$ & 0.1136725 & 0.1136725 & $|1,2,2\rangle$ & $1 d_{3 / 2}$ & 0.1136725 & 0.1136725 \\
\hline$|1,3,-4\rangle$ & $1 f_{7 / 2}$ & 0.1438029 & 0.1438031 & $|1,3,3\rangle$ & $1 f_{5 / 2}$ & 0.1438029 & 0.1438031 \\
\hline$|1,4,-5\rangle$ & $1 g_{9 / 2}$ & 0.1791421 & 0.1791425 & $|1,4,4\rangle$ & $1 g_{7 / 2}$ & 0.1791421 & 0.1791425 \\
\hline
\end{tabular}

TABLE 9: The bound state energy eigenvalues for the Hulthen potential in the pseudospin symmetric case for $m=5 \mathrm{fm}^{-1}$ and $S_{p s}=-5 \mathrm{fm}^{-1}$. Here first states column is according to $(l, j)$ and second states column is according to $(l+2, j+1)$.

\begin{tabular}{|c|c|c|c|c|c|c|c|}
\hline$|n, \widetilde{l}, \kappa\rangle$ & State & $E_{n \kappa}$ & $E_{n \kappa}[40]$ & $|n-1, \tilde{l}, \kappa\rangle$ & State & $E_{n \kappa}$ & $E_{n \kappa}[40]$ \\
\hline$|1,1,-1\rangle$ & $1 s_{1 / 2}$ & -0.00875024 & -0.00862755 & $|0,1,2\rangle$ & $0 d_{3 / 2}$ & -0.00875024 & -0.00862755 \\
\hline$|1,2,-2\rangle$ & $1 p_{3 / 2}$ & -0.01566113 & -0.01538462 & $|0,2,3\rangle$ & $0 f_{5 / 2}$ & -0.01566113 & -0.01538462 \\
\hline$|1,3,-3\rangle$ & $1 d_{5 / 2}$ & -0.02467877 & -0.02413384 & $|0,3,4\rangle$ & $0 g_{7 / 2}$ & -0.02467877 & -0.02413384 \\
\hline$|1,4,-4\rangle$ & $1 f_{7 / 2}$ & -0.03708404 & -0.03492388 & $|0,4,5\rangle$ & $0 h_{9 / 2}$ & -0.03708404 & -0.03492388 \\
\hline$|2,1,-1\rangle$ & $2 s_{1 / 2}$ & -0.01554783 & -0.01538462 & $|1,1,2\rangle$ & $1 d_{3 / 2}$ & -0.01554783 & -0.01538462 \\
\hline$|2,2,-2\rangle$ & $2 p_{3 / 2}$ & -0.02452379 & -0.02413384 & $|1,2,3\rangle$ & $1 f_{5 / 2}$ & -0.02452379 & -0.02413384 \\
\hline$|2,3,-3\rangle$ & $2 d_{5 / 2}$ & -0.03574590 & -0.03492388 & $|1,3,4\rangle$ & $1 g_{7 / 2}$ & -0.03574590 & -0.03492388 \\
\hline$|2,4,-4\rangle$ & $2 f_{7 / 2}$ & -0.04888397 & -0.04781723 & $|1,4,5\rangle$ & $1 h_{9 / 2}$ & -0.04888397 & -0.04781723 \\
\hline
\end{tabular}

TABLE 10: The bound state energy eigenvalues for the Hulthen potential in the spin symmetric case for $m=5 \mathrm{fm}^{-1}$ and $S_{s}=5 \mathrm{fm}^{-1}$. Here first and second states columns are according to $(l, j)$.

\begin{tabular}{|c|c|c|c|c|c|c|c|}
\hline$|n, l, \kappa\rangle$ & State & $E_{n \kappa}$ & $E_{n \kappa}[40]$ & $|n, l, \kappa\rangle$ & State & $E_{n \kappa}$ & $E_{n \kappa}[40]$ \\
\hline$|0,1,-2\rangle$ & $0 p_{3 / 2}$ & 0.06582980 & 0.06784763 & $|0,1,1\rangle$ & $0 p_{1 / 2}$ & 0.06582980 & 0.06784763 \\
\hline$|0,2,-3\rangle$ & $0 d_{5 / 2}$ & 0.14592114 & 0.15074992 & $|0,2,2\rangle$ & $0 d_{3 / 2}$ & 0.14592114 & 0.15074992 \\
\hline$|0,3,-4\rangle$ & $0 f_{7 / 2}$ & 0.25504870 & 0.26338976 & $|0,3,3\rangle$ & $0 f_{5 / 2}$ & 0.25504870 & 0.26338976 \\
\hline$|0,4,-5\rangle$ & $0 g_{9 / 2}$ & 0.39036621 & 0.40263365 & $|0,4,4\rangle$ & $0 g_{7 / 2}$ & 0.39036621 & 0.40263365 \\
\hline$|1,1,-2\rangle$ & $1 p_{3 / 2}$ & 0.14708189 & 0.15074992 & $|1,1,1\rangle$ & $1 p_{1 / 2}$ & 0.14708189 & 0.15074992 \\
\hline$|1,2,-3\rangle$ & $1 d_{5 / 2}$ & 0.25580818 & 0.26338976 & $|1,2,2\rangle$ & $1 d_{3 / 2}$ & 0.25580818 & 0.26338976 \\
\hline$|1,3,-4\rangle$ & $1 f_{7 / 2}$ & 0.39073879 & 0.40263365 & $|1,3,3\rangle$ & $1 f_{5 / 2}$ & 0.39073879 & 0.40263365 \\
\hline$|1,4,-5\rangle$ & $1 g_{9 / 2}$ & 0.54849660 & 0.56482502 & $|1,4,4\rangle$ & $1 g_{7 / 2}$ & 0.54849660 & 0.56482502 \\
\hline
\end{tabular}

TABLE 11: The bound state energy eigenvalues for the Yukawa potential in the pseudospin symmetric case for $m=5 \mathrm{fm}^{-1}$ and $S_{p s}=-5 \mathrm{fm}{ }^{-1}$. Here first states column is according to $(l, j)$ and second states column is according to $(l+2, j+1)$.

\begin{tabular}{|c|c|c|c|c|c|c|c|}
\hline$|n, \widetilde{l}, \kappa\rangle$ & State & $E_{n \kappa}$ & $E_{n \kappa}[55,56]$ & $|n-1, \widetilde{l}, \kappa\rangle$ & State & $E_{n \kappa}$ & $E_{n \kappa}[55,56]$ \\
\hline$|1,1,-1\rangle$ & $1 s_{1 / 2}$ & -0.0184387 & - & $|0,1,2\rangle$ & $0 d_{3 / 2}$ & -0.0184387 & -0.0184387 \\
\hline$|1,2,-2\rangle$ & $1 p_{3 / 2}$ & -0.0327981 & -0.0020419 & $|0,2,3\rangle$ & $0 f_{5 / 2}$ & -0.0327981 & -0.0328771 \\
\hline$|1,3,-3\rangle$ & $1 d_{5 / 2}$ & -0.0515637 & -0.0081778 & $|0,3,4\rangle$ & $0 g_{7 / 2}$ & -0.0515637 & -0.0515686 \\
\hline$|1,4,-4\rangle$ & $1 f_{7 / 2}$ & -0.0746132 & -0.0184387 & $|0,4,5\rangle$ & $0 h_{9 / 2}$ & -0.0746132 & -0.0746134 \\
\hline$|2,1,-1\rangle$ & $2 s_{1 / 2}$ & -0.0327981 & -0.0020419 & $|1,1,2\rangle$ & $1 d_{3 / 2}$ & -0.0327981 & -0.0328771 \\
\hline$|2,2,-2\rangle$ & $2 p_{3 / 2}$ & -0.0515686 & - & $|1,2,3\rangle$ & $1 f_{5 / 2}$ & -0.0515686 & -0.0515686 \\
\hline$|2,3,-3\rangle$ & $2 d_{5 / 2}$ & -0.0744240 & -0.0020419 & $|1,3,4\rangle$ & $1 g_{7 / 2}$ & -0.0744240 & -0.0746134 \\
\hline$|2,4,-4\rangle$ & $2 f_{7 / 2}$ & -0.1021320 & -0.0081778 & $|1,4,5\rangle$ & $1 h_{9 / 2}$ & -0.1021320 & -0.1021400 \\
\hline
\end{tabular}


TABLE 12: The bound state energy eigenvalues for the Yukawa potential in the spin symmetric case for $m=5 \mathrm{fm}^{-1}$ and $S_{s}=5 \mathrm{fm}^{-1}$. Here first and second states columns are according to $(l, j)$.

\begin{tabular}{|c|c|c|c|c|c|c|c|}
\hline$|n, l, \kappa\rangle$ & State & $E_{n \kappa}$ & $E_{n \kappa}[56]$ & $|n, l, \kappa\rangle$ & State & $E_{n \kappa}$ & $E_{n \kappa}[56]$ \\
\hline$|0,1,-2\rangle$ & $0 p_{3 / 2}$ & 0.0078560 & 0.0019617 & $|0,1,1\rangle$ & $0 p_{1 / 2}$ & 0.0078560 & 0.0078560 \\
\hline$|0,2,-3\rangle$ & $0 d_{5 / 2}$ & 0.0177103 & 0.0078560 & $|0,2,2\rangle$ & $0 d_{3 / 2}$ & 0.0177103 & 0.0177103 \\
\hline$|0,3,-4\rangle$ & $0 f_{7 / 2}$ & 0.0315710 & 0.0177103 & $|0,3,3\rangle$ & $0 f_{5 / 2}$ & 0.0315710 & 0.0315710 \\
\hline$|0,4,-5\rangle$ & $0 g_{9 / 2}$ & 0.0495050 & 0.0315710 & $|0,4,4\rangle$ & $0 g_{7 / 2}$ & 0.0495050 & 0.0495050 \\
\hline$|1,1,-2\rangle$ & $1 p_{3 / 2}$ & 0.0177103 & - & $|1,1,1\rangle$ & $1 p_{1 / 2}$ & 0.0177103 & 0.0177103 \\
\hline$|1,2,-3\rangle$ & $1 d_{5 / 2}$ & 0.0314949 & 0.0019617 & $|1,2,2\rangle$ & $1 d_{3 / 2}$ & 0.0314949 & 0.0315710 \\
\hline$|1,3,-4\rangle$ & $1 f_{7 / 2}$ & 0.0495002 & 0.0078560 & $|1,3,3\rangle$ & $1 f_{5 / 2}$ & 0.0495002 & 0.0495050 \\
\hline$|1,4,-5\rangle$ & $1 g_{9 / 2}$ & 0.0716001 & 0.0177103 & $|1,4,4\rangle$ & $1 g_{7 / 2}$ & 0.0716001 & 0.0716004 \\
\hline
\end{tabular}

TABLE 13: The bound state energy eigenvalues for the Woods-Saxon potential in the pseudospin symmetric case for $m=5 \mathrm{fm}^{-1}$ and $S_{p s}=0 \mathrm{fm}^{-1}$. Here first states column is according to $(l, j)$ and second states column is according to $(l+2, j+1)$.

\begin{tabular}{lccccc}
\hline$|n, \tilde{l}, \kappa\rangle$ & State & $E_{n \kappa}$ & $|n, \tilde{l}, \kappa\rangle$ & State & $E_{n \kappa}$ \\
\hline$|1,1,-1\rangle$ & $1 s_{1 / 2}$ & -4.97891 & $|0,1,2\rangle$ & $0 d_{3 / 2}$ & -4.97891 \\
$|1,2,-2\rangle$ & $1 p_{3 / 2}$ & -4.98562 & $|0,2,3\rangle$ & $0 f_{5 / 2}$ & -4.98562 \\
$|1,3,-3\rangle$ & $1 d_{5 / 2}$ & -4.99564 & $|0,3,4\rangle$ & $0 g_{7 / 2}$ & -4.99564 \\
$|1,4,-4\rangle$ & $1 f_{7 / 2}$ & -5.00894 & $|0,4,5\rangle$ & $0 h_{9 / 2}$ & -5.00894 \\
$|2,1,-1\rangle$ & $2 s_{1 / 2}$ & -4.81296 & $|1,1,2\rangle$ & $1 d_{3 / 2}$ & -4.81296 \\
$|2,2,-2\rangle$ & $2 p_{3 / 2}$ & -4.81873 & $|1,2,3\rangle$ & $1 f_{5 / 2}$ & -4.81873 \\
$|2,3,-3\rangle$ & $2 d_{5 / 2}$ & -4.82742 & $|1,3,4\rangle$ & $1 g_{7 / 2}$ & -4.82742 \\
$|2,4,-4\rangle$ & $2 f_{7 / 2}$ & -4.83904 & $|1,4,5\rangle$ & $1 h_{9 / 2}$ & -4.83904 \\
\hline
\end{tabular}

symmetry, bound energy eigenvalues have not achieved. However, we find bound energy eigenvalues for $1 s_{1 / 2}, 1 p_{3 / 2}$, and $2 p_{3 / 2}$ states and also we obtain pseudospin and spin doublets. The reason of this is the approximation. Unlike $[55,56]$ we use the Pekeris approximation to the centrifugal term.

7.6. Woods-Saxon Potential. If the potential parameters are taken $A=0, q=p=1, B=-V_{0}, b=1 / a$, and $L=R$, the generalized Manning-Rosen potential reduces to the WoodsSaxon potential [83]:

$$
V_{\mathrm{WS}}(r)=-\frac{V_{0}}{1+e^{(r-R) / a}},
$$

where $a$ is thickness of surface and $R$ is nuclear radius.

For this potential, the bound energy eigenvalues are found in pseudospin and spin symmetric cases by using (44) and (37) for $b=2 \mathrm{fm}^{-1}, L=7 \mathrm{fm}, m=5 \mathrm{fm}^{-1}, B=-2 \mathrm{fm}^{-1}$, and $S_{s}=S_{p s}=0$. These energy eigenvalues are presented in Tables 13 and 14. Also for the other set of parameters like $m=10 \mathrm{fm}^{-1}, B=-10 \mathrm{fm}^{-1}, b=2 \mathrm{fm}^{-1}, L=7 \mathrm{fm}$, and $S_{p s}=$ $-10.1 \mathrm{fm}^{-1}$, our energy eigenvalues for pseudospin symmetry which can be found by using (44) are same as energy eigenvalues of [35] that is obtained in the absence of tensor potential.

7.7. q-Parameter Hyperbolic Pöschl-Teller Potential. q-parameter hyperbolic Pöschl-Teller potential [79] is obtained by
TABLE 14: The bound state energy eigenvalues for the Woods-Saxon potential in the spin symmetric case for $m=5 \mathrm{fm}^{-1}$ and $S_{s}=0 \mathrm{fm}^{-1}$. Here first and second states columns are according to $(l, j)$.

\begin{tabular}{lccccc}
\hline$|n, l, \kappa\rangle$ & State & $E_{n \kappa}$ & $|n, l, \kappa\rangle$ & State & $E_{n \kappa}$ \\
\hline$|0,1,-2\rangle$ & $0 p_{3 / 2}$ & 2.10339 & $|0,1,1\rangle$ & $0 p_{1 / 2}$ & 2.10339 \\
$|0,2,-3\rangle$ & $0 d_{5 / 2}$ & 2.13554 & $|0,2,2\rangle$ & $0 d_{3 / 2}$ & 2.13554 \\
$|0,3,-4\rangle$ & $0 f_{7 / 2}$ & 2.18283 & $|0,3,3\rangle$ & $0 f_{5 / 2}$ & 2.18283 \\
$|0,4,-5\rangle$ & $0 g_{9 / 2}$ & 2.24422 & $|0,4,4\rangle$ & $0 g_{7 / 2}$ & 2.24422 \\
$|1,1,-2\rangle$ & $1 p_{3 / 2}$ & 3.00686 & $|1,1,1\rangle$ & $1 p_{1 / 2}$ & 3.00686 \\
$|1,2,-3\rangle$ & $1 d_{5 / 2}$ & 3.02052 & $|1,2,2\rangle$ & $1 d_{3 / 2}$ & 3.02052 \\
$|1,3,-4\rangle$ & $1 f_{7 / 2}$ & 3.04086 & $|1,3,3\rangle$ & $1 f_{5 / 2}$ & 3.04086 \\
$|1,4,-5\rangle$ & $1 g_{9 / 2}$ & 3.06769 & $|1,4,4\rangle$ & $1 g_{7 / 2}$ & 3.06769 \\
\hline
\end{tabular}

taking the parameters as $p=\widetilde{q}, A=4 V_{0} \widetilde{q}, B=-4 V_{0}, b=2 \alpha$, and $q=e^{2 \alpha L}$ in (1):

$$
V_{q \mathrm{HPT}}(r)=-V_{0} \frac{4}{\left(e^{\alpha r}+\widetilde{q} e^{-\alpha r}\right)^{2}}=-\frac{V_{0}}{\cosh _{\tilde{q}}^{2}(\alpha r)} .
$$

For this potential, we find energy eigenvalues from (44) in pseudospin symmetric limit and from (37) in spin symmetric limit by considering $V_{0}=2, b=0.2, L=7, m=5, S_{s}=5$, and $S_{p s}=-5$. These energy eigenvalues are given in Tables 15 and 16 for pseudospin and spin symmetric limits, respectively.

\section{Conclusion}

In the present study, spin and pseudospin symmetric Dirac spinors have been obtained for a new suggested generalized Manning-Rosen potential by solving the Dirac equation with centrifugal term. In order to solve the Dirac equation, the Nikiforov-Uvarov method has been used and the Pekeris approximation has been applied to the centrifugal term. Besides, we have obtained energy eigenvalues equations for potential considered in this study in both spin and pseudospin symmetry cases and by using these energy equations, binding energies of some states are calculated for a special set of parameters. The obtained results are given in Tables 1 and 2. It is seen from these tables that the binding energies are taken negative values for pseudospin symmetry case and positive values for spin symmetry limit. In addition we have investigated the effects of potential parameters $b, q, L$, and $p$ 
TABLE 15: The bound state energy eigenvalues for the q-parameter hyperbolic Pöschl-Teller potential in the pseudospin symmetric case for $m=5 \mathrm{fm}^{-1}$ and $S_{p s}=-5 \mathrm{fm}^{-1}$. Here first states column is according to $(l, j)$ and second states column is according to $(l+2, j+1)$.

\begin{tabular}{|c|c|c|c|c|c|c|c|}
\hline$|n, \tilde{l}, \kappa\rangle$ & State & $\frac{(\widetilde{q}=1)}{E_{n \kappa}}$ & $\frac{(\widetilde{q}=2)}{E_{n \kappa}}$ & $|n, \widetilde{l}, \kappa\rangle$ & State & $\frac{(\widetilde{q}=1)}{E_{n \kappa}}$ & $\frac{(\widetilde{q}=2)}{E_{n \kappa}}$ \\
\hline$|1,1,-1\rangle$ & $1 s_{1 / 2}$ & -0.216038 & -0.270379 & $|0,1,2\rangle$ & $0 d_{3 / 2}$ & -0.216038 & -0.270379 \\
\hline$|1,2,-2\rangle$ & $1 p_{3 / 2}$ & -0.608149 & -0.803145 & $|0,2,3\rangle$ & $0 f_{5 / 2}$ & -0.608149 & -0.803145 \\
\hline$|1,3,-3\rangle$ & $1 d_{5 / 2}$ & -1.160371 & -1.577490 & $|0,3,4\rangle$ & $0 g_{7 / 2}$ & -1.160371 & -1.577490 \\
\hline$|1,4,-4\rangle$ & $1 f_{7 / 2}$ & -1.843543 & -2.566032 & $|0,4,5\rangle$ & $0 h_{9 / 2}$ & -1.843543 & -2.566032 \\
\hline$|2,1,-1\rangle$ & $2 s_{1 / 2}$ & -0.223653 & -0.255494 & $|1,1,2\rangle$ & $1 d_{3 / 2}$ & -0.223653 & -0.255494 \\
\hline$|2,2,-2\rangle$ & $2 p_{3 / 2}$ & -0.639701 & -0.807669 & $|1,2,3\rangle$ & $1 f_{5 / 2}$ & -0.639701 & -0.807669 \\
\hline$|2,3,-3\rangle$ & $2 d_{5 / 2}$ & -1.218299 & -1.606114 & $|1,3,4\rangle$ & $1 g_{7 / 2}$ & -1.218299 & -1.606114 \\
\hline$|2,4,-4\rangle$ & $2 f_{7 / 2}$ & -1.929906 & -2.626000 & $|1,4,5\rangle$ & $1 h_{9 / 2}$ & -1.929906 & -2.626000 \\
\hline
\end{tabular}

TABLE 16: The bound state energy eigenvalues for the q-parameter hyperbolic Pöschl-Teller potential in the spin symmetric case for $m=5 \mathrm{fm}^{-1}$ and $S_{s}=5 \mathrm{fm}^{-1}$. Here first and second states columns are according to $(l, j)$.

\begin{tabular}{|c|c|c|c|c|c|c|c|}
\hline$|n, l, \kappa\rangle$ & State & $\frac{(\widetilde{q}=1)}{E_{n \kappa}}$ & $\frac{(\widetilde{q}=2)}{E_{n \kappa}}$ & $|n, l, \kappa\rangle$ & State & $\frac{(\widetilde{q}=1)}{E_{n \kappa}}$ & $\frac{(\widetilde{q}=2)}{E_{n \kappa}}$ \\
\hline$|0,1,-2\rangle$ & $0 p_{3 / 2}$ & 3.173391 & 4.077032 & $|0,1,1\rangle$ & $0 p_{1 / 2}$ & 3.173391 & 4.077032 \\
\hline$|0,2,-3\rangle$ & $0 d_{5 / 2}$ & 3.321749 & 4.125918 & $|0,2,2\rangle$ & $0 d_{3 / 2}$ & 3.321749 & 4.125918 \\
\hline$|0,3,-4\rangle$ & $0 f_{7 / 2}$ & 3.484972 & 4.185197 & $|0,3,3\rangle$ & $0 f_{5 / 2}$ & 3.484972 & 4.185197 \\
\hline$|0,4,-5\rangle$ & $0 g_{9 / 2}$ & 3.642339 & 4.248060 & $|0,4,4\rangle$ & $0 g_{7 / 2}$ & 3.642339 & 4.248060 \\
\hline$|1,1,-2\rangle$ & $1 p_{3 / 2}$ & 3.321462 & 4.171962 & $|1,1,1\rangle$ & $1 p_{1 / 2}$ & 3.321462 & 4.171962 \\
\hline$|1,2,-3\rangle$ & $1 d_{5 / 2}$ & 3.468183 & 4.224145 & $|1,2,2\rangle$ & $1 d_{3 / 2}$ & 3.468183 & 4.224145 \\
\hline$|1,3,-4\rangle$ & $1 f_{7 / 2}$ & 3.630026 & 4.287317 & $|1,3,3\rangle$ & $1 f_{5 / 2}$ & 3.630026 & 4.287317 \\
\hline$|1,4,-5\rangle$ & $1 g_{9 / 2}$ & 3.786248 & 4.354272 & $|1,4,4\rangle$ & $1 g_{7 / 2}$ & 3.786248 & 4.354272 \\
\hline
\end{tabular}

and parameters $m, S_{s}$, and $S_{p s}$ on the binding energies of some states both for spin and pseudospin symmetry in Figures 2, $3,4,5,6$, and 7 . The figures show that the binding energies increase as $m, S_{s}$ for spin symmetry and $m, S_{p s}$ for pseudospin symmetry increase and decrease as $p$ and $L$ increase in both symmetry cases. However unlike parameter $q$, the binding energies increase for spin symmetry and decrease for pseudospin symmetry as parameter $b$ increases. Moreover, special cases of the generalized Manning-Rosen potential which are Manning-Rosen, Hulthen, Woods-Saxon, Morse, Kratzer-Fues, Yukawa, and q-parameter hyperbolic PöschlTeller potentials have been examined. Energy eigenvalues for these potentials have been found in spin and pseudospin symmetry cases. Also these energy eigenvalues have been compared with energy eigenvalues of the previous studies and it is seen that our results are consistent with the previous ones.

The other important results of this study have been summarized in the following.

(i) A new suggested generalized Manning-Rosen potential is considered as a nuclear potential because this potential can be reduced to Woods-Saxon, Hulthen, and Yukawa potentials which are nuclear potentials. At the same time the suggested potential in this study is regarded as a molecular potential too because it can be reduced to Manning-Rosen, Morse, Kratzer-Fues, and hyperbolic Pöschl-Teller potentials which are used to explain molecular structure.

(ii) The energy eigenvalues that are obtained for Hulthen potentials have been compared with the results of [40] in
Tables 9 and 10 and it is seen that our results are little different from the results of [40]. The reason of the difference is form of the approximation which is applied to the centrifugal term. The authors of [40] have used the Greene-Aldrich approximation while we have used the Pekeris approximation. Also the obtained results for Yukawa potential have been compared with the results of $[55,56]$ in Tables 11 and 12 . The results of $[55,56]$ in which the Greene-Aldrich approximation is used show that bound energy eigenvalues do not exist for some states and the members of spin and pseudospin doublets do not have the same energies but our results indicate that bound energy eigenvalues exist in all states and members of spin and pseudospin doublets have the same energies. Explanation of this situation is that our Pekeris approximation is more convenient according to the Greene-Aldrich approximation for the centrifugal term.

(iii) We have used the Nikiforov-Uvarov method to solve Dirac equation, while in [49] standard method, in [27] SUSYQM, and in [46] asymptotic iteration method have been used. Nevertheless, our results are consistent with [27, 46, 49] as seen in Tables 3, 5, 6, 7, and 8. Therefore, it can be concluded that the methods which are used to solve Dirac equation do not affect the results.

\section{Conflict of Interests}

The authors declare that there is no conflict of interests regarding the publication of this paper. 


\section{Acknowledgments}

The authors wish to thank Dr. Oktay Aydoğdu, Dr. Kenan Sogut, and referees for helpful suggestions and comments.

\section{References}

[1] J. N. Ginocchio, "Relativistic symmetries in nuclei and hadrons," Physics Reports, vol. 414, no. 4-5, pp. 165-261, 2005.

[2] J. N. Ginocchio, "Relativistic harmonic oscillator with spin symmetry," Physical Review -Nuclear Physics, vol. 69, no. 3, Article ID 034318, 2004.

[3] S. G. Zhou, J. Meng, and P. Ring, "Spin symmetry in the antinucleon spectrum," Physical Review Letters, vol. 91, no. 26, Article ID 262501, 4 pages, 2003.

[4] J. N. Ginocchio, "A relativistic symmetry in nuclei," Physics Report, vol. 315, no. 1-4, pp. 231-240, 1999.

[5] P. R. Page, T. Goldman, and J. N. Ginocchio, "Relativistic symmetry suppresses quark spin-orbit splitting," Physical Review Letters, vol. 86, article 204, 2001.

[6] K. T. Hecht and A. Adler, "Generalized seniority for favored $J \neq$ 0 pairs in mixed configurations," Nuclear Physics A, vol. 137, no. 1, pp. 129-143, 1969.

[7] A. Arima, M. Harvey, and K. Shimizu, "Pseudo LS coupling and pseudo SU3 coupling schemes," Physics Letters B, vol. 30, no. 8, pp. 517-522, 1969.

[8] A. Bohr, I. Hamamoto, and B. R. Mottelson, "Pseudospin in rotating nuclear potentials," Physica Scripta, vol. 26, no. 4, pp. 267-272, 1982.

[9] W. Nazarewicz, P. J. Twin, P. Fallon, and J. D. Garrett, "Naturalparity states in superdeformed bands and pseudo SU(3) symmetry at extreme conditions," Physical Review Letters, vol. 64, no. 14, pp. 1654-1657, 1990.

[10] F. S. Stephens, M. A. Deleplanque, J. E. Draper et al., "Pseudospin symmetry and quantized alignment in nuclei," Physical Review Letters, vol. 65, article 301, 1990.

[11] B. Mottelson, "Some themes in the study of very deformed rotating nuclei," Nuclear Physics, Section A, vol. 522, no. 1-2, pp. $1-12,1991$.

[12] D. Troltenier, W. Nazarewicz, Z. Szymański, and J. P. Draayer, "On the validity of the pseudo-spin concept for axially symmetric deformed nuclei," Nuclear Physics, Section A, vol. 567, no. 3, pp. 591-610, 1994.

[13] A. E. Stuchbery, "Magnetic behaviour in the pseudo-Nilsson model," Journal of Physics G: Nuclear and Particle Physics, vol. 25, no. 4, pp. 611-615, 1999.

[14] A. E. Stuchbery, "Magnetic properties of rotational states in the pseudo-Nilsson model," Nuclear Physics A, vol. 700, no. 1-2, pp. 83-116, 2002.

[15] D. Troltenier, C. Bahri, and J. P. Draayer, "Generalized pseudoSU(3) model and pairing," Nuclear Physics A, vol. 586, no. 1, pp. 53-72, 1995.

[16] C. Bahri, J. P. Draayer, and S. A. Moszkowski, "Pseudospin symmetry in nuclear physics," Physical Review Letters, vol. 68, article 2133, 1992.

[17] A. L. Blokhin, C. Bahri, and J. P. Draayer, "Origin of pseudospin symmetry," Physical Review Letters, vol. 74, no. 21, pp. 41494152, 1995.

[18] A. L. Blokhin, C. Bahri, and J. P. Draayer, "Pseudospin transformation of physical operators," Journal of Physics A, vol. 29, no. 9, pp. 2039-2052, 1996.
[19] J. N. Ginocchio, "Pseudospin as a relativistic symmetry," Physical Review Letters, vol. 78, no. 3, pp. 436-439, 1997.

[20] J. N. Ginocchio and A. Leviatan, "On the relativistic foundations of pseudospin symmetry in nuclei," Physics Letters B, vol. 425, no. 1-2, pp. 1-5, 1998.

[21] J. N. Ginocchio, "On the relativisitic origins of pseudo-spin symmetry in nuclei," Journal of Physics G: Nuclear and Particle Physics, vol. 25, no. 4, article 617, 1999.

[22] A. Leviatan and J. N. Ginocchio, "Consequences of a relativistic pseudospin symmetry for radial nodes and intruder levels in nuclei," Physics Letters, Section B: Nuclear, Elementary Particle and High-Energy Physics, vol. 518, no. 1-2, pp. 214-220, 2001.

[23] J. Meng, K. Sugawara-Tanabe, S. Yamaji, P. Ring, and A. Arima, "Pseudospin symmetry in relativistic mean field theory," Physical Review C - Nuclear Physics, vol. 58, no. 2, pp. R628R631, 1998.

[24] J. Meng, K. Sugawara-Tanabe, S. Yamaji, and A. Arima, "Pseudospin symmetry in $\mathrm{Zr}$ and $\mathrm{Sn}$ isotopes from the proton drip line to the neutron drip line," Physical Review C: Nuclear Physics, vol. 59, no. 1, pp. 154-163, 1999.

[25] J. S. Bell and H. Ruegg, "Dirac equations with an exact higher symmetry," Nuclear Physics B, vol. 98, no. 1, pp. 151-153, 1975.

[26] G.-F. Wei and S.-H. Dong, "Approximately analytical solutions of the Manning-Rosen potential with the spin-orbit coupling term and spin symmetry," Physics Letters A, vol. 373, no. 1, pp. 49-53, 2008.

[27] G.-F. Wei and S.-H. Dong, "Pseudospin symmetry in the relativistic Manning-Rosen potential including a Pekeris-type approximation to the pseudo-centrifugal term," Physics Letters B, vol. 686, no. 4-5, pp. 288-292, 2010.

[28] T. Chen, J.-Y. Liu, and C.-S. Jia, "Approximate analytical solutions of the Dirac-Manning-Rosen problem with the spin symmetry and pseudo-spin symmetry," Physica Scripta, vol. 79, no. 5, Article ID 055002, 2009.

[29] F. Taşkın, "Approximate solutions of the Dirac equation for the Manning-Rosen potential including the spin-orbit coupling term," International Journal of Theoretical Physics, vol. 48, no. 4, pp. 1142-1149, 2009.

[30] H. Hassanabadi, E. Maghsoodi, S. Zarrinkamar, and H. Rahimov, "Actual and general manning-Rosen potentials under spin and pseudospin symmetries of the Dirac equation," Canadian Journal of Physics, vol. 90, no. 7, pp. 633-646, 2012.

[31] S. Asgarifar and H. Goudarzi, "Exact solutions of the ManningRosen potential plus a ring-shaped like potential for the Dirac equation: spin and pseudospin symmetry," Physica Scripta, vol. 87, no. 2, Article ID 025703, 2013.

[32] H. Feizi and A. H. Ranjbar, "Relativistic symmetries of the Manning-Rosen potential in the frame of supersymmetry," The European Physical Journal Plus, vol. 128, article 3, 2013.

[33] H. Tokmehdashi, A. A. Rajabi, and M. Hamzavi, "Hulthén and coulomb-like potentials as a tensor interaction within the relativistic symmetries of the manning-rosen potential," Advances in High Energy Physics, vol. 2014, Article ID 870523, 14 pages, 2014.

[34] S. Ortakaya, H. Hassanabadi, and E. Maghsoodi, "Scattering phase shifts of Dirac equation with Manning-Rosen potential and Yukawa tensor interaction," Indian Journal of Physics, vol. 89, no. 4, pp. 307-316, 2015.

[35] O. Aydoğdu and R. Sever, "Pseudospin and spin symmetry in the Dirac equation with Woods-Saxon potential and tensor potential," The European Physical Journal A, vol. 43, no. 1, pp. 73-81, 2010. 
[36] E. Maghsoodi, H. Hassanabadi, S. Zarrinkamar, and H. Rahimov, "Relativistic symmetries of the Dirac equation under the nuclear WoodsSaxon potential," Physica Scripta, vol. 85, no. 5, Article ID 055007, 2012.

[37] S. M. Ikhdair and R. Sever, "Approximate analytical solutions of the generalized Woods-Saxon potentials including the spinorbit coupling term and spin symmetry," Central European Journal of Physics, vol. 8, no. 4, pp. 652-666, 2010.

[38] J.-Y. Guo and Z.-Q. Sheng, "Solution of the Dirac equation for the Woods-Saxon potential with spin and pseudospin symmetry," Physics Letters A, vol. 338, no. 2, pp. 90-96, 2005.

[39] H. Feizi, M. R. Shojaei, and A. A. Rajabi, "Raising and lowering operators for the Dirac-Woods-Saxon potential in the presence of spin and pseudospin symmetry," The European Physical Journal Plus, vol. 127, article 41, 2012.

[40] O. Aydoğdu, E. Maghsoodi, and H. Hassanabadi, "Dirac equation for the Hulthén potential within the Yukawa-type tensor interaction," Chinese Physics B, vol. 22, no. 1, Article ID 010302, 2013.

[41] S. M. Ikhdair, C. Berkdemir, and R. Sever, "Spin and pseudospin symmetry along with orbital dependency of the Dirac-Hulthén problem," Applied Mathematics and Computation, vol. 217, no. 22, pp. 9019-9032, 2011.

[42] A. Soylu, O. Bayrak, and I. Boztosun, "An approximate solution of Dirac-Hulthén problem with pseudospin and spin symmetry for any $\kappa$ state," Journal of Mathematical Physics, vol. 48, Article ID 082302, 2007.

[43] A. N. Ikot, H. Hassanabadi, E. Maghsoodi, and S. Zarrinkamar, "Relativistic symmetries of Hulthén potential incorporated with generalized tensor interactions," Advances in High Energy Physics, vol. 2013, Article ID 910419, 10 pages, 2013.

[44] S. M. Ikhdair and R. Sever, "Approximate bound state solutions of Dirac equation with Hulthén potential including Coulomblike tensor potential," Applied Mathematics and Computation, vol. 216, no. 3, pp. 911-923, 2010.

[45] O. Aydoğdu and R. Sever, "Pseudospin and spin symmetry for the ring-shaped generalized Hulthén potential," International Journal of Modern Physics A: Particles and Fields. Gravitation; Cosmology, vol. 25, no. 21, pp. 4067-4079, 2010.

[46] O. Aydoğdu and R. Sever, "Exact solution of the Dirac equation with the Mie-type potential under the pseudospin and spin symmetry limit," Annals of Physics, vol. 325, no. 2, pp. 373-383, 2010.

[47] M. Hamzavi, A. A. Rajabi, and H. Hassanabadi, "Exact spin and pseudospin symmetry solutions of the Dirac equation for Mietype potential including a Coulomb-like tensor potential," FewBody Systems, vol. 48, no. 2-4, pp. 171-182, 2010.

[48] M. Eshghi and S. M. Ikhdair, "Relativistic effect of pseudospin symmetry and tensor coupling on the Mie-type potential via Laplace transformation method," Chinese Physics B, vol. 23, no. 12, article 120304, 2014.

[49] O. Aydoğdu and R. Sever, "Pseudospin and spin symmetry in Dirac-Morse problem with a tensor potential," Physics Letters B, vol. 703, no. 3, pp. 379-385, 2011.

[50] O. Bayrak and I. Boztosun, "The pseudospin symmetric solution of the Morse potential for any $\kappa$ state," Journal of Physics A: Mathematical and Theoretical, vol. 40, no. 36, pp. 11119-11127, 2007.

[51] C. Berkdemir, "Pseudospin symmetry in the relativistic Morse potential including the spin-orbit coupling term," Nuclear Physics A, vol. 770, no. 1-2, pp. 32-39, 2006.
[52] W.-C. Qiang, R.-S. Zhou, and Y. Gao, "Application of the exact quantization rule to the relativistic solution of the rotational Morse potential with pseudospin symmetry," Journal of Physics A. Mathematical and Theoretical, vol. 40, no. 7, pp. 1677-1685, 2007.

[53] L.-H. Zhang, X.-P. Li, and C.-S. Jia, "Approximate analytical solutions of the Dirac equation with the generalized Morse potential model in the presence of the spin symmetry and pseudo-spin symmetry," Physica Scripta, vol. 80, no. 3, Article ID 035003, 2009.

[54] S. M. Ikhdair, "An approximate $\kappa$ state solutions of the Dirac equation for the generalized Morse potential under spin and pseudospin symmetry," Journal of Mathematical Physics, vol. 52, Article ID 052303, 2011.

[55] O. Aydoğdu and R. Sever, "The Dirac-Yukawa problem in view of pseudospin symmetry," Physica Scripta, vol. 84, no. 2, Article ID 025005, 2011.

[56] E. Maghsoodi, H. Hassanabadi, and O. Aydoğdu, "Dirac particles in the presence of the Yukawa potential plus a tensor interaction in SUSYQM framework," Physica Scripta, vol. 86, no. 1, Article ID 015005, 2012.

[57] S. M. Ikhdair, "Approximate $\kappa$-state solutions to the DiracYukawa problem based on the spin and pseudospin symmetry," Central European Journal of Physics, vol. 10, no. 2, pp. 361-381, 2012.

[58] M. R. Setare and S. Haidari, "Spin symmetry of the Dirac equation with the Yukawa potential," Physica Scripta, vol. 81, no. 6, Article ID 065201, 2010.

[59] A. Arda and R. Sever, "Approximate analytical solutions of the Dirac equation for Yukawa potential plus tensor interaction with any $\kappa$-value," Few-Body Systems, vol. 54, no. 11, pp. 18291837, 2013.

[60] X.-Y. Liu, G.-F. Wei, X.-W. Cao, and H.-G. Bai, "Spin symmetry for Dirac equation with the trigonometric Pöschl-Teller potential," International Journal of Theoretical Physics, vol. 49, no. 2, pp. 343-348, 2010.

[61] C.-S. Jia, P. Guo, Y.-F. Diao, L.-Z. Yi, and X.-J. Xie, "Solutions of Dirac equations with the Pöschl-Teller potential," European Physical Journal A, vol. 34, no. 1, pp. 41-48, 2007.

[62] Y. Xu, S. He, and C.-S. Jia, "Approximate analytical solutions of the Dirac equation with the Pöschl-Teller potential including the spin-orbit coupling term," Journal of Physics A: Mathematical and Theoretical, vol. 41, no. 25, Article ID 255302, 2008.

[63] C.-S. Jia, T. Chen, and L.-G. Cui, "Approximate analytical solutions of the Dirac equation with the generalized PöschlTeller potential including the pseudo-centrifugal term," Physics Letters A, vol. 373, no. 18-19, pp. 1621-1626, 2009.

[64] G.-F. Wei and S.-H. Dong, "The spin symmetry for deformed generalized Pöschl-Teller potential," Physics Letters A, vol. 373, no. 29, pp. 2428-2431, 2009.

[65] G. F. Wei and S. H. Dong, "Algebraic approach to pseudospin symmetry for the Dirac equation with scalar and vector modified Pöschl-Teller potentials," EPL (Europhysics Letters), vol. 87, Article ID 40004, 2009.

[66] G.-F. Wei and S.-H. Dong, "A novel algebraic approach to spin symmetry for Dirac equation with scalar and vector second Pöschl-Teller potentials," The European Physical Journal A, vol. 43, no. 2, pp. 185-190, 2010.

[67] A. Taş, S. Alpdoğan, and A. Havare, "The scattering and bound states of the Schrödinger particle in generalized asymmetric manning-rosen type potential," Advances in High Energy Physics, vol. 2014, Article ID 619241, 10 pages, 2014. 
[68] M. F. Manning and N. Rosen, "A potential function for the vibrations of diatomic molecules," Physical Review, vol. 44, no. 11, pp. 953-954, 1933.

[69] W.-C. Qiang and S.-H. Dong, "Analytical approximations to the solutions of the Manning-Rosen potential with centrifugal term," Physics Letters A, vol. 368, no. 1-2, pp. 13-17, 2007.

[70] W.-C. Qiang and S.-H. Dong, "The Manning-Rosen potential studied by a new approximate scheme to the centrifugal term," Physica Scripta, vol. 79, no. 4, Article ID 045004, 2009.

[71] S.-H. Dong and J. García-Ravelo, "Exact solutions of the s-wave Schrödinger equation with Manning-Rosen potential," Physica Scripta, vol. 75, no. 3, pp. 307-309, 2007.

[72] G.-F. Wei, C.-Y. Long, and S.-H. Dong, "The scattering of the Manning-Rosen potential with centrifugal term," Physics Letters A, vol. 372, no. 15, pp. 2592-2596, 2008.

[73] X.-Y. Gu and S.-H. Dong, "Energy spectrum of the ManningRosen potential including centrifugal term solved by exact and proper quantization rules," Journal of Mathematical Chemistry, vol. 49, no. 9, pp. 2053-2062, 2011.

[74] P. M. Morse, "Diatomic molecules according to the wave mechanics. II. Vibrational levels," Physical Review, vol. 34, no. 1, pp. 57-64, 1929.

[75] S.-H. Dong and G.-H. Sun, "The series solutions of the nonrelativistic equation with the Morse potential," Physics Letters. A, vol. 314, no. 4, pp. 261-266, 2003.

[76] W.-C. Qiang and S.-H. Dong, "Arbitrary $l$-state solutions of the rotating Morse potential through the exact quantization rule method," Physics Letters A, vol. 363, no. 3, pp. 169-176, 2007.

[77] J. Yu, S.-H. Dong, and G.-H. Sun, "Series solutions of the Schrödinger equation with position-dependent mass for the Morse potential," Physics Letters A, vol. 322, no. 5-6, pp. 290$297,2004$.

[78] G. Pöschl and E. Teller, "Bemerkungen zur Quantenmechanik des anharmonischen Oszillators," Zeitschrift für Physik, vol. 83, no. 3-4, pp. 143-151, 1933.

[79] H. Yanar, A. Havare, and K. Sogut, "Scattering and bound states of Duffin-Kemmer-Petiau particles for $q$-parameter hyperbolic Pöschl-Teller potential," Advances in High Energy Physics, vol. 2014, Article ID 840907, 9 pages, 2014.

[80] A. Kratzer, "Die ultraroten Rotationsspektren der Halogenwasserstoffe," Zeitschrift für Physik, vol. 3, no. 5, pp. 289-307, 1920.

[81] E. Fues, "Das Eigenschwingungsspektrum zweiatomiger Moleküle in der Undulationsmechanik," Annalen der Physik, vol. 385, no. 12, pp. 367-396, 1926.

[82] O. Bayrak, I. Boztosun, and H. Ciftci, "Exact analytical solutions to the kratzer potential by the asymptotic iteration method," International Journal of Quantum Chemistry, vol. 107, no. 3, pp. 540-544, 2007.

[83] R. D. Woods and D. S. Saxon, "Diffuse surface optical model for nucleon-nuclei scattering," Physical Review, vol. 95, no. 2, pp. 577-578, 1954.

[84] L. Hulthen, "Uber die Eigenlösungen der Schrödinger chung des Deutrons," Arkiv för Matematik, Astronomi och Fysik, vol. 28A, no. 5, 1942.

[85] H. Yukawa, "The prediction and discovery of pions and muons," Proceedings of the Physico-Mathematical Society of Japan, vol. 17, p. $48,1935$.

[86] A. F. Nikiforov and V. B. Uvarov, Special Functions of Mathematical Physics, Birkhäuser, Basel, Switzerland, 1988.
[87] W. Greiner, Relativistic Quantum Mechanics, Springer, Berlin, Germany, 2nd edition, 1997.

[88] J. J. Sakurai and J. Napolitano, Modern Quantum Mechanics, Addison-Wesley, Boston, Mass, USA, 2nd edition, 2010.

[89] C. L. Pekeris, "The rotation-vibration coupling in diatomic molecules," Physical Review, vol. 45, article 98, 1934.

[90] K. Sogut and A. Havare, "Transmission resonances in the Duffin-Kemmer-Petiau equation in $(1+1)$ dimensions for an asymmetric cusp potential," Physica Scripta, vol. 82, no. 4, Article ID 045013, 2010.

[91] R. L. Greene and C. Aldrich, "Variational wave functions for a screened Coulomb potential," Physical Review A, vol. 14, no. 6, pp. 2363-2366, 1976. 

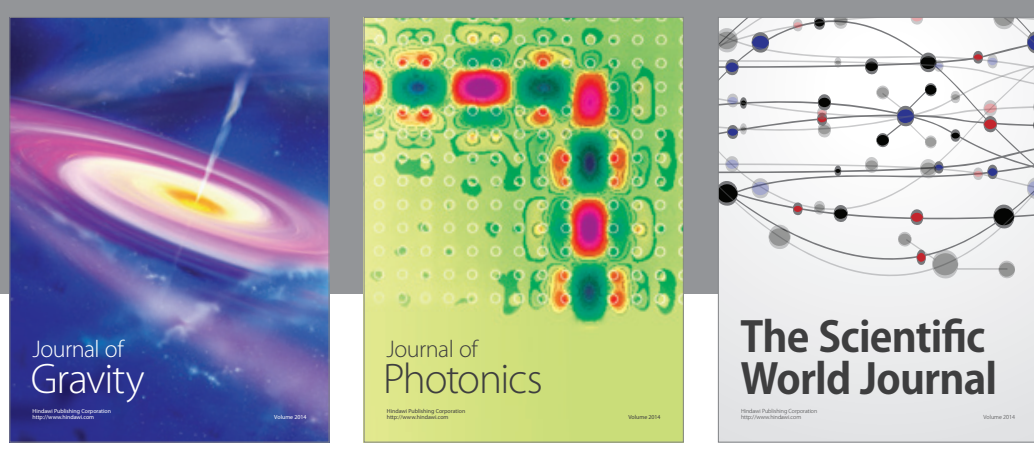

The Scientific World Journal
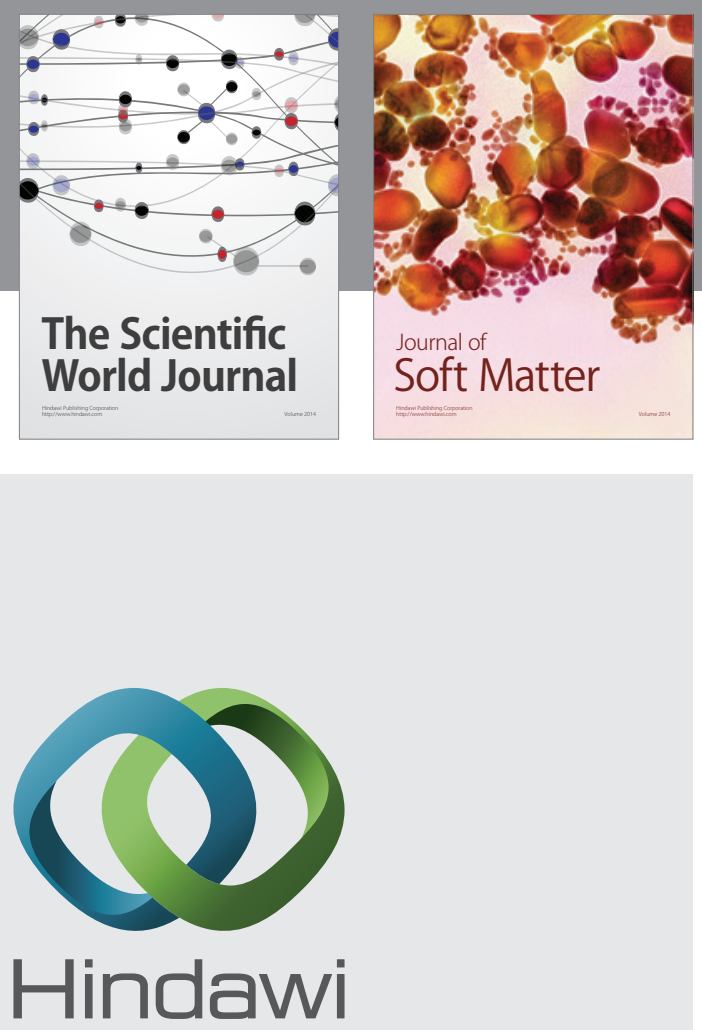

Submit your manuscripts at

http://www.hindawi.com

nternational Journal of

Statistical Mechanics
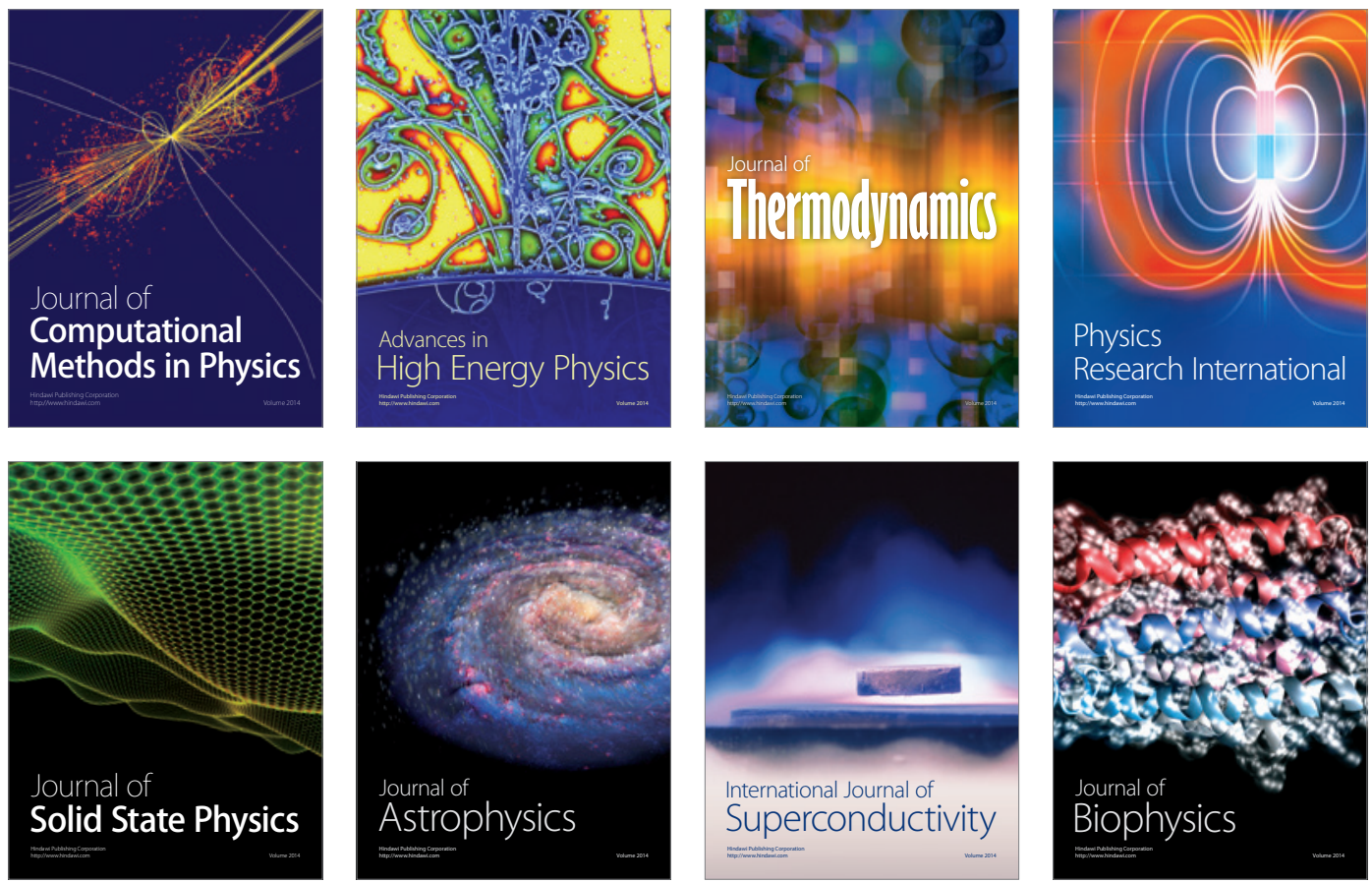
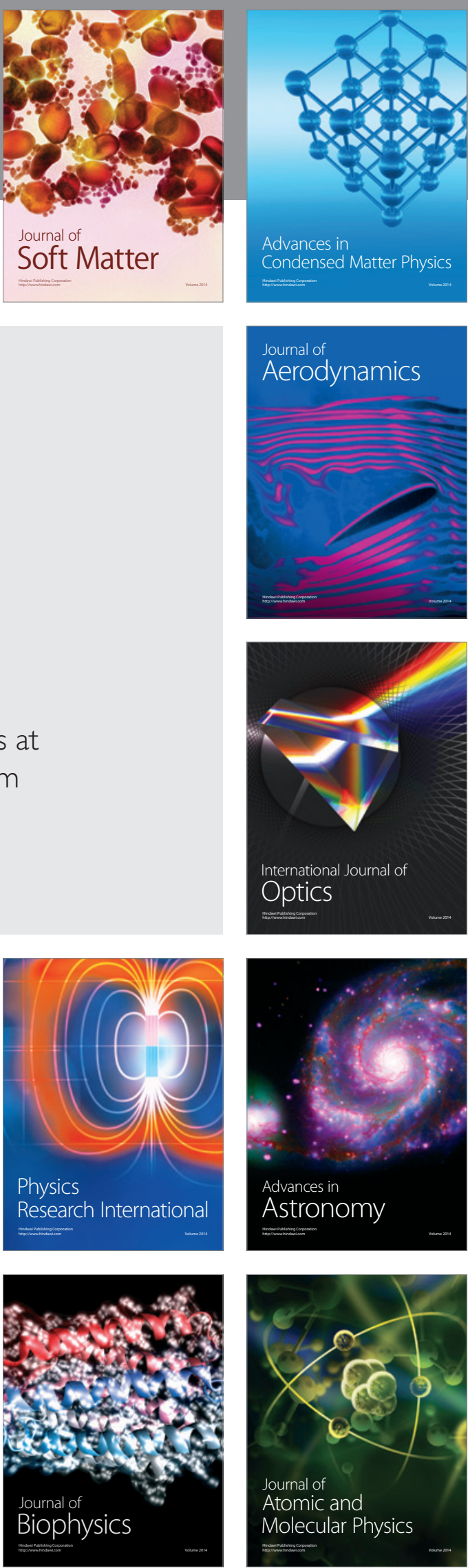